\title{
GLOBAL CARLEMAN INEQUALITIES FOR PARABOLIC SYSTEMS AND APPLICATIONS TO CONTROLLABILITY*
}

\author{
ENRIQUE FERNÁNDEZ-CARA ${ }^{\dagger}$ AND SERGIO GUERRERO ${ }^{\dagger}$
}

\begin{abstract}
This paper has been conceived as an overview on the controllability properties of some relevant (linear and nonlinear) parabolic systems. Specifically, we deal with the null controllability and the exact controllability to the trajectories. We try to explain the role played by the observability inequalities in this context and the need of global Carleman estimates. We also recall the main ideas used to overcome the difficulties motivated by nonlinearities. First, we considered the classical heat equation with Dirichlet conditions and distributed controls. Then we analyze recent extensions to other linear and semilinear parabolic systems and/or boundary controls. Finally, we review the controllability properties for the Stokes and Navier-Stokes equations that are known to date. In this context, we have paid special attention to obtaining the necessary Carleman estimates. Some open questions are mentioned throughout the paper. We hope that this unified presentation will be useful for those researchers interested in the field.
\end{abstract}

Key words. Carleman inequalities, parabolic equations, unique continuation, observability, null controllability

AMS subject classifications. 35B37, 70Q05, 93B05, 93B07

DOI. $10.1137 / \mathrm{S} 0363012904439696$

Introduction. The goal of this paper is to provide a panorama of an important subfield of control theory: the null controllability analysis of parabolic equations and systems.

The main contributions to this area are due to O. Yu. Imanuvilov, who popularized the use of global Carleman estimates in the context of null controllability. Many arguments from [36], [30], [39], and [37] will be reproduced here. Another relevant contributor has been E. Zuazua, who was able to deduce global controllability results for some nonlinear systems for the first time in [56].

The controllability of partial differential equations has been the object of intensive research during the last few decades. In 1978, Russell [53] made a survey of the most relevant results that were available in the literature at that time. In that paper, the author described a number of different tools that were developed to address controllability problems, often inspired and related to other subjects concerning partial differential equations: multipliers, moment problems, nonharmonic Fourier series, etc. More recently, J.-L. Lions introduced the so-called Hilbert uniqueness method (for instance, see [44], [45], [46]). That was the starting point of a fruitful period on the subject.

It would be impossible to present here all the relevant results that have been proved in this area. We will thus reduce our scope drastically, considering only null controllability problems for parabolic equations and systems.

In order to get an idea, let us consider the simplest case of the linear heat equation

\footnotetext{
* Received by the editors January 14, 2004; accepted for publication (in revised form) April 10, 2006; published electronically October 12, 2006. This work was partially supported by D.G.E.S. (Spain), grant BFM2003-06446.

http://www.siam.org/journals/sicon/45-4/43969.html

†Departamento de Ecuaciones Diferenciales y Análisis Numérico, Universidad de Sevilla, Aptdo. 1160, 41080 Sevilla, Spain (cara@numer.us.es, sguerrero@us.es).
} 
with Dirichlet boundary conditions and distributed controls:

$$
\begin{cases}y_{t}-\Delta y=v 1_{\mathcal{O}} & \text { in } \quad Q=\Omega \times(0, T), \\ y=0 & \text { on } \quad \Sigma=\partial \Omega \times(0, T), \\ y(0)=y^{0} & \text { in } \quad \Omega .\end{cases}
$$

Here, $\Omega \subset \mathbf{R}^{N}$ is a bounded domain of class $C^{2}, \mathcal{O} \subset \Omega$ is a nonempty open subset, $1_{\mathcal{O}}$ is the characteristic function of $\mathcal{O}$, and $T$ is a given positive time. We assume that the initial state $y^{0}$ is given in $L^{2}(\Omega)$ and try to find a control $v \in L^{2}(\mathcal{O} \times(0, T))$ such that the associated state $y=y(x, t)$ possesses a desired behavior at time $t=T$.

Roughly speaking, systems of parabolic type like (0.1) are characterized by nonreversibility, the dissipativity of the solutions (i.e., the fact that energy is lost along the trajectories), and the regularizing effect.

In accordance with this last property, it is not possible to lead the solutions of (0.1) exactly to every final state in a Sobolev space like $L^{2}(\Omega)$ or $H^{m}(\Omega)$, unless the control is exerted brutally, i.e., unless we are in the trivial and uninteresting situation $\mathcal{O}=\Omega$.

On the contrary, it may be interesting to investigate whether it is possible to drive the solutions to $(0.1)$ (acting on a small set $\mathcal{O} \subset \subset \Omega$ ) exactly to a state on a trajectory. For instance, it is completely meaningful to search for controls $v$ such that the associated states $y$ satisfy

$$
y(T)=0 \quad \text { in } \quad \Omega .
$$

If such controls exist, we say that (0.1) is null controllable at time $T$.

Observe that the null controllability of a parabolic system is a very useful property from the viewpoint of applications. Indeed, it permits us to reach in a finite amount of time a state that is "natural" for the system. All the work has to be done for $t \in[0, T]$. Afterwards, no additional effort is needed to get a satisfactory situation.

It is also easy to see that, for a linear system of the kind above, null controllability is equivalent to exact controllability to the trajectories, i.e., to the following property: for any $y^{0} \in L^{2}(\Omega)$ and any function $\bar{y}$ satisfying

$$
\begin{cases}\bar{y}_{t}-\Delta \bar{y}=0 & \text { in } \quad Q, \\ \bar{y}=0 & \text { on } \quad \Sigma\end{cases}
$$

and

$$
\bar{y} \in C^{0}\left([0, T] ; L^{2}(\Omega)\right)
$$

there exist controls $v$ such that

$$
y(T)=\bar{y}(T) \quad \text { in } \quad \Omega .
$$

It will be explained below that the null controllability of a linear parabolic system is, roughly speaking, equivalent to the observability of the associated adjoint states.

More precisely, for each $\varphi^{0} \in L^{2}(\Omega)$, let us consider the so-called adjoint system

$$
\begin{cases}-\varphi_{t}-\Delta \varphi=0 & \text { in } Q, \\ \varphi=0 & \text { on } \Sigma, \\ \varphi(T)=\varphi^{0} & \text { in } \Omega .\end{cases}
$$


Then (0.1) is null controllable with controls in $L^{2}(\mathcal{O} \times(0, T))$ if and only if there exists $C>0$ such that

$$
\|\varphi(0)\|_{L^{2}(\Omega)}^{2} \leq C \iint_{\mathcal{O} \times(0, T)}|\varphi|^{2} d x d t \quad \forall \varphi^{0} \in L^{2}(\Omega) .
$$

At present, the most powerful tools to prove inequalities like (0.4) for general parabolic systems are global Carleman estimates. They have the form

$$
\iint_{\Omega \times(0, T)} \rho^{2}|\varphi|^{2} d x d t \leq C \iint_{\mathcal{O} \times(0, T)} \rho^{2}|\varphi|^{2} d x d t
$$

where $\rho=\rho(x, t)$ is continuous and strictly positive for $t \in(0, T)$. The previous considerations motivate the need for establishing such estimates.

It is also natural to ask if null controllability and/or exact controllability to the trajectories hold in the framework of semilinear or even genuinely nonlinear parabolic systems. For instance, we can be interested in controls and states satisfying

$$
\begin{cases}y_{t}-\Delta y+f(y)=v 1_{\mathcal{O}} & \text { in } Q, \\ y=0 & \text { on } \Sigma, \\ y(0)=y^{0} & \text { in } \Omega\end{cases}
$$

and (0.2), where the function $f: \mathbf{R} \mapsto \mathbf{R}$ is given and, for instance, we assume that $f \in C^{1}(\mathbf{R})$ and $f(0)=0$.

For systems of this kind, a classical approach relies on the use of fixed point arguments. The idea is to find a fixed point of the mapping $z \mapsto y$, where $y$ is, together with some $v$, a solution to the linearized system

$$
\begin{cases}y_{t}-\Delta y+\frac{f(z)}{z} y=v 1_{\mathcal{O}} & \text { in } Q, \\ y=0 & \text { on } \Sigma, \\ y(0)=y^{0} & \text { in } \Omega\end{cases}
$$

satisfying (0.2). The existence of $v$ is again equivalent to an observability inequality which is implied by appropriate Carleman estimates similar to (0.5).

The fixed point approach can be applied whenever, among other things, the way the constants arising in Carleman estimates depend on the coefficients of the linearized systems is known in detail. Therefore, a careful analysis of (0.5) is needed.

The theoretical results we present below can be applied in many contexts. For instance, they can serve as tools for

- controlling the temperature of a medium eventually influenced by transport and/or chemical effects;

- controlling the velocity field of a real fluid (like those modeled by the NavierStokes equations), etc.

In the following sections, we will explain how these ideas can be applied to several parabolic equations and systems. A similar (but different) overview on this topic is [6], where the author is also concerned with the stabilization of parabolic systems.

An important aspect of the controllability of time-dependent systems that will not be treated in this paper is the analysis and obtainment of numerical approximations. Of course, the most interesting question concerning the null controllability of (0.1) and (0.6) is how to construct a control with the desirable properties. 
Several ideas that reproduce the theoretical arguments at a finite-dimensional level were given, for instance, in [34]. Unfortunately, the proposed algorithms seem to be still too expensive. We simply point out here that the controllability analysis we recall in section 2 , based on the solution of a linear fourth order problem, could provide some promising ideas.

There are many other important subjects related to the controllability of parabolic equations that will not be visited here. Let us mention some of them:

- The analysis of the existence of insensitizing controls in the sense of [47] or, more generally, the null controllability of cascade systems. This has been the objective of considerable work in recent years (see, for instance, [12], [9], and $[26])$.

- The controllability of parabolic systems with memory. This includes interesting families of linear viscoelastic fluids. Some partial results in this field are given in [7] and [14].

- The control of coupled systems of parabolic-hyperbolic type, like a combination of heat and wave equations, the system of thermoelasticity, etc. Some of these problems have been treated in [57], [42], and [43].

- The control of fluid-solid interaction systems. This seems to be unexplored at present. For very simple models, some results are given in [13].

- The control of nondeterministic systems. Some advances were made in the context of linear problems. See, for instance, [8]. In this field, the main open question turns out to be the obtainment of controllability results for nonlinear systems, due to the lack of compactness.

The plan of this paper is the following. In section 1, we consider the classical heat equation and some variants. Essentially, we deal there with heat equations with strong solutions. We recall the proof of a basic Carleman estimate and present some applications to semilinear systems.

Section 2 is devoted to the null controllability analysis of other more complicated parabolic equations involving weak and/or very weak solutions. It will be shown that the proof of the related Carleman inequalities is much more involved and needs some extra work. We will also indicate how this can be used to deduce the exact controllability to the trajectories of more general semilinear systems.

Finally, section 3 is concerned with systems of the Stokes kind. Here, the main difficulties are caused by the pressure. We will recall a recent argument leading to an appropriate but not completely satisfactory Carleman inequality. We will also explain how this leads to the null controllability of Stokes-like systems and to the local exact controllability to the trajectories of the Navier-Stokes equations.

\section{Null controllability of the linear heat equation and applications.}

1.1. Null controllability and observability. Let us consider again the simplest case of the linear heat equation with Dirichlet boundary conditions and distributed control with support in a small set:

$$
\begin{cases}y_{t}-\Delta y=v 1_{\mathcal{O}} & \text { in } \quad Q, \\ y=0 & \text { on } \Sigma, \\ y(0)=y^{0} & \text { in } \Omega .\end{cases}
$$

We assume that $\mathcal{O} \subset \subset \Omega$ is a nonempty (small) open subset and $T$ is a given positive time. In what follows, $n(x)$ will stand for the outward unit normal vector at the point $x \in \partial \Omega$. We assume that the initial state $y^{0}$ is given in $L^{2}(\Omega)$, and we try to 
find a control $v \in L^{2}(\mathcal{O} \times(0, T))$ such that the associated state $y=y(x, t)$ possesses a desired behavior at time $t=T$.

Recall that under these assumptions, system (1.1) has a unique weak solution $y$ satisfying

$$
y \in L^{2}\left(0, T ; H_{0}^{1}(\Omega)\right) \cap C^{0}\left([0, T] ; L^{2}(\Omega)\right)
$$

that depends continuously on $y^{0}$ and $v$.

Our interest is to provide an answer to the following questions.

Question 1: Null controllability. For every $y^{0} \in L^{2}(\Omega)$, can a control $v \in L^{2}(\mathcal{O} \times$ $(0, T))$ be found such that $y(T)=0$ in $\Omega$ ?

This question will be answered affirmatively in this section. To this end, we will introduce, for each $\varphi^{0} \in L^{2}(\Omega)$, the adjoint system

$$
\begin{cases}-\varphi_{t}-\Delta \varphi=0 & \text { in } Q, \\ \varphi=0 & \text { on } \Sigma, \\ \varphi(T)=\varphi^{0} & \text { in } \Omega,\end{cases}
$$

and we will try to answer the following auxiliary question.

Question 2: Observability inequality. Can we find a constant $C>0$ such that, for each $\varphi^{0} \in L^{2}(\Omega)$, the associated solution of (1.2) satisfies

$$
\|\varphi(0)\|_{L^{2}(\Omega)}^{2} \leq C \iint_{\mathcal{O} \times(0, T)}|\varphi|^{2} d x d t ?
$$

An affirmative answer to Question 2 implies an affirmative answer to Question 1. This is what is proved in the following result.

THEOREM 1.1. The observability inequality (1.3) implies the null controllability of (1.1).

Proof. We divide the proof into two steps. First, we build a sequence of controls $v_{\varepsilon} \in L^{2}(\mathcal{O} \times(0, T))$ with $\varepsilon>0$ which provide the approximate controllability of (1.1) (see (1.7)). Second, we pass to the limit when $\varepsilon$ tends to zero and we conclude.

Step 1 . Let $y^{0} \in L^{2}(\Omega)$ and $\varepsilon>0$ be given. Let us introduce the functional $J_{\varepsilon}$, with

$$
J_{\varepsilon}\left(\varphi^{0}\right)=\frac{1}{2} \iint_{\mathcal{O} \times(0, T)}|\varphi|^{2} d x d t+\varepsilon\left\|\varphi^{0}\right\|_{L^{2}(\Omega)}+\left(\varphi(0), y^{0}\right)_{L^{2}(\Omega)}
$$

for every $\varphi^{0} \in L^{2}(\Omega)$. Here, $\varphi$ is the solution of (1.2) associated to the initial condition $\varphi^{0}$. Using (1.3), it is not difficult to check that $J_{\varepsilon}$ is strictly convex, continuous, and coercive in $L^{2}(\Omega)$, so it possesses a unique minimum $\varphi_{\varepsilon}^{0} \in L^{2}(\Omega)$, whose associated solution is denoted by $\varphi_{\varepsilon}$. Let us now introduce the control $v_{\varepsilon}=\varphi_{\varepsilon} 1_{\mathcal{O}}$, and let us denote by $y_{\varepsilon}$ the solution of (1.1) associated to $v_{\varepsilon}$.

Let $y_{1}$ be the final state of the solution to (1.1) with vanishing control. Let us remark that the unique interesting case to be studied turns out to be when $\left\|y_{1}\right\|_{L^{2}(\Omega)}>$ $\varepsilon$, since this is equivalent to $\varphi_{\varepsilon}^{0} \neq 0$. See [19] for more details. Under this assumption, we can differentiate the functional $J_{\varepsilon}$ at $\varphi_{\varepsilon}^{0}$ and obtain a necessary condition for $J_{\varepsilon}$ to reach a minimum at $\varphi_{\varepsilon}^{0}$, say,

$$
\iint_{\mathcal{O} \times(0, T)} \varphi_{\varepsilon} \varphi d x d t+\varepsilon\left(\frac{\varphi_{\varepsilon}^{0}}{\left\|\varphi_{\varepsilon}^{0}\right\|_{L^{2}(\Omega)}}, \varphi^{0}\right)_{L^{2}(\Omega)}+\left(\varphi(0), y^{0}\right)_{L^{2}(\Omega)}=0
$$

for every $\varphi^{0} \in L^{2}(\Omega)$. 
Using this and (1.3) for $\varphi^{0}=\varphi_{\varepsilon}^{0}$, we obtain $\left\|v_{\varepsilon}\right\|_{L^{2}(\mathcal{O} \times(0, T))} \leq \sqrt{C}\left\|y^{0}\right\|_{L^{2}(\Omega)}$, where $C$ is the observability constant of (1.3).

Since systems (1.1) and (1.2) are in duality, we have

$$
\iint_{\mathcal{O} \times(0, T)} \varphi_{\varepsilon} \varphi d x d t=\left(y_{\varepsilon}(T), \varphi^{0}\right)_{L^{2}(\Omega)}-\left(y^{0}, \varphi(0)\right)_{L^{2}(\Omega)},
$$

which, combined with (1.5), yields

$$
\left\|y_{\varepsilon}(T)\right\|_{L^{2}(\Omega)} \leq \varepsilon
$$

Step 2. Since the sequence $\left\{v_{\varepsilon}\right\}$ is bounded in $L^{2}(\mathcal{O} \times(0, T))$, it possesses a (weakly) convergent subsequence to certain $v \in L^{2}(\mathcal{O} \times(0, T))$. Using classical parabolic estimates we deduce that, at least for a subsequence,

$$
y_{\varepsilon} \rightarrow y \quad \text { weakly in } L^{2}\left(0, T ; H_{0}^{1}(\Omega)\right) \cap H^{1}\left(0, T ; H^{-1}(\Omega)\right),
$$

where $y$ is the solution of (1.1) with control $v$. In particular, this gives weak convergence for $\left\{y_{\varepsilon}(t)\right\}(t \in[0, T])$ in $L^{2}(\Omega)$ so we have $y(T)=0$.

Remark 1 . At this point, some comments must be made:

1. We have proved that (1.3) implies null controllability with a control that satisfies

$$
\|v\|_{L^{2}(\mathcal{O} \times(0, T))} \leq \sqrt{C}\left\|y^{0}\right\|_{L^{2}(\Omega)},
$$

where $C$ is the observability constant.

Conversely, if we have null controllability with controls $v \in L^{2}(\mathcal{O} \times(0, T))$ that satisfy

$$
\|v\|_{L^{2}(\mathcal{O} \times(0, T))} \leq \sqrt{C}\left\|y^{0}\right\|_{L^{2}(\Omega)}
$$

for some constant $C>0$, then it can be checked that we have (1.3) with the same constant $C$.

2. It is possible to present a similar argument in a general frame. Let us consider three Hilbert spaces $U, H, E$ and two linear continuous operators $L \in \mathcal{L}(U ; E)$ and $M \in \mathcal{L}(H ; E)$. Then we have

$$
\left\|M^{*} \varphi^{0}\right\|_{H} \leq C\left\|L^{*} \varphi^{0}\right\|_{U^{\prime}} \quad \forall \varphi^{0} \in E^{\prime}
$$

for some positive constant $C$ if and only if $R(M) \subset R(L)$ and, moreover,

$$
\forall y^{0} \in H, \exists v \in U \text { such that } L v=M y^{0},\|v\|_{U} \leq C\left\|y^{0}\right\|_{H} .
$$

Properties of this kind have been established and analyzed for the first time in the framework of control theory in [52]. They have been successfully used in many different contexts in recent years.

3. Because of linearity, the null controllability of (1.1) is equivalent to the exact controllability to (uncontrolled) trajectories. In other words, (1.1) is null controllable if and only if, for every $\bar{y}^{0} \in L^{2}(\Omega)$, we can find a control $v \in$ $L^{2}(\mathcal{O} \times(0, T))$ such that $y(T)=\bar{y}(T)$ in $\Omega$, where $y$ is the solution of (1.1) associated to $v$, and $\bar{y}$ satisfies

$$
\begin{cases}\bar{y}_{t}-\Delta \bar{y}=0 & \text { in } \quad Q, \\ \bar{y}=0 & \text { on } \quad \Sigma, \\ \bar{y}(0)=\bar{y}^{0} & \text { in } \quad \Omega .\end{cases}
$$


4. In general, for any $y^{1} \in L^{2}(\Omega)$, it is not reasonable to look for a control $v \in L^{2}(\mathcal{O} \times(0, T))$ such that $y(T)=y^{1}$ in $\Omega$ (recall that $\mathcal{O} \subset \subset \Omega$ ). Indeed, due to the regularizing effect, every solution to (1.1) is space-analytic in $\Omega \backslash \overline{\mathcal{O}}$ at time $T$, which is not necessarily the case for $y^{1}$. Thus, the exact controllability problem is out of order for the heat equation.

In what follows, for the reasons stated above, we will focus on the proof of (1.3). This will rely on other (previous) inequalities for the solutions to (1.2), known as global Carleman inequalities. Let us mention that a general global Carleman inequality adopts the form

$$
\iint_{\Omega \times(0, T)} \rho^{2}|\varphi|^{2} d x d t \leq C \iint_{\mathcal{O} \times(0, T)} \rho^{2}|\varphi|^{2} d x d t,
$$

where $\rho=\rho(x, t)$ is a continuous and strictly positive weight function. For a function $\rho$ satisfying $\rho>0$ in $\bar{\Omega} \times(0, T)$, we will be able to deduce (1.14) and then estimates like

$$
\iint_{\Omega \times(T / 4,3 T / 4)}|\varphi|^{2} d x d t \leq C \iint_{\mathcal{O} \times(0, T)}|\varphi|^{2} d x d t
$$

This, together with the dissipation properties of the solutions of (1.2), will lead to (1.3). More details are given below.

Remark 2. To our knowledge, the first (boundary) controllability results for the heat equation were obtained by Egorov in the early sixties; see [17]. Later, an important general principle was established by Russell in [52]: if $T_{0}$ is sufficiently large and the wave system

$$
\begin{cases}y_{t t}-\Delta y=v 1_{\mathcal{O}} & \text { in } \Omega \times\left(0, T_{0}\right), \\ y=0 & \text { on } \partial \Omega \times\left(0, T_{0}\right), \\ y(0)=y^{0}, \quad y_{t}(0)=y^{1} & \text { in } \Omega\end{cases}
$$

is exactly controllable at time $T_{0}$, then (1.1) is null controllable at time $T$ for all $T>0$.

This principle has been revisited by several authors. In particular, an "abstract" version is given in [4].

Using the method of moments, the exact controllability of (1.16) can be established for large $T_{0}$ when, for instance, $\overline{\mathcal{O}}$ is a neighborhood of $\partial \Omega$. Thus, one has null controllability for (1.1) in this case.

Remark 3. In the particular case of the classical heat equation (1.1), there is another way to prove (1.3), based on the spectral decomposition of the solutions. This approach was introduced by Lebeau and Robbiano [41] and led to the null controllability of the heat equation with controls supported by general subdomains $\mathcal{O} \subset \subset \Omega$ for the first time.

1.2. A global Carleman inequality for the linear heat equation and its consequences.

Lemma 1.2. Let $\omega \subset \subset \Omega$ be a nonempty open subset. Then there exists $\eta^{0} \in$ $C^{2}(\bar{\Omega})$ such that $\eta^{0}>0$ in $\Omega, \eta^{0}=0$ on $\partial \Omega$, and $\left|\nabla \eta^{0}\right|>0$ in $\overline{\Omega \backslash \omega}$.

A proof of this lemma can be found in [30]. A much easier proof can be developed when $\Omega$ is star shaped with respect to a point $x^{0} \in \omega$. 
Let $\omega$ be a nonempty open set satisfying $\omega \subset \subset \mathcal{O}$ and let us set

$$
\begin{aligned}
\alpha(x, t) & =\frac{e^{2 \lambda m\left\|\eta^{0}\right\|_{\infty}}-e^{\lambda\left(m\left\|\eta^{0}\right\|_{\infty}+\eta^{0}(x)\right)}}{t(T-t)}, \\
\xi(x, t) & =\frac{e^{\lambda\left(m\left\|\eta^{0}\right\|_{\infty}+\eta^{0}(x)\right)}}{t(T-t)}
\end{aligned}
$$

for $(x, t) \in Q$, where $\eta^{0}$ is the function furnished by Lemma 1.2 for this $\omega$ and $m>1$. Weight functions of this kind were first introduced by Imanuvilov. See [30] for a systematic use of them.

Now we arrive at the main result of this section.

LEMma 1.3. There exist three constants $\lambda_{1}=C(\Omega, \mathcal{O}) \geq 1, s_{1}=C(\Omega, \mathcal{O})$ $\left(T+T^{2}\right)$, and $C_{1}(\Omega, \mathcal{O})$ such that, for any $\lambda \geq \lambda_{1}$ and any $s \geq s_{1}$, the following inequality holds:

$$
\begin{aligned}
& s^{-1} \iint_{Q} e^{-2 s \alpha} \xi^{-1}\left(\left|q_{t}\right|^{2}+|\Delta q|^{2}\right) d x d t+s \lambda^{2} \iint_{Q} e^{-2 s \alpha} \xi|\nabla q|^{2} d x d t \\
& \quad+s^{3} \lambda^{4} \iint_{Q} e^{-2 s \alpha} \xi^{3}|q|^{2} d x d t \leq C_{1}\left(\iint_{Q} e^{-2 s \alpha}\left|q_{t}+\Delta q\right|^{2} d x d t\right. \\
& \left.\quad+s^{3} \lambda^{4} \iint_{\mathcal{O} \times(0, T)} e^{-2 s \alpha} \xi^{3}|q|^{2} d x d t\right)
\end{aligned}
$$

for all $q \in C^{2}(\bar{Q})$ with $q=0$ on $\Sigma$.

In what follows, $C(\Omega, \mathcal{O})$ or simply $C$ will denote a generic constant depending only on $\Omega$ and $\mathcal{O}$.

Before giving the proof of Lemma 1.3, we will deduce the observability inequality (1.3) (and, accordingly, the null controllability of (1.1)) from this result. This can be made in three steps.

Step 1. From the density of the smooth functions in the space where the solutions of $(1.2)$ with $\varphi^{0} \in L^{2}(\Omega)$ live, we first observe that the Carleman inequality above is verified by all of them. Thus, fixing $\lambda=\lambda_{1}$, we get

$$
\iint_{Q} e^{-2 s \alpha} t^{-3}(T-t)^{-3}|\varphi|^{2} d x d t \leq C \iint_{\mathcal{O} \times(0, T)} e^{-2 s \alpha} t^{-3}(T-t)^{-3}|\varphi|^{2} d x d t
$$

for all $s \geq s_{1}$.

Step 2. Using the inequalities

$$
e^{-2 s_{1} \alpha} t^{-3}(T-t)^{-3} \geq e^{-2 C(\Omega, \mathcal{O})(1+1 / T)} \frac{1}{T^{6}} \quad \text { in } \quad \Omega \times(T / 4,3 T / 4)
$$

and

$$
e^{-2 s_{1} \alpha} t^{-3}(T-t)^{-3} \leq e^{-C(\Omega, \mathcal{O})(1+1 / T)} \frac{1}{T^{6}} \quad \text { in } \quad \Omega \times(0, T),
$$

we get

$$
\iint_{\Omega \times(T / 4,3 T / 4)}|\varphi|^{2} d x d t \leq C(\Omega, \mathcal{O}, T) \iint_{\mathcal{O} \times(0, T)}|\varphi|^{2} d x d t
$$


with a constant $C(\Omega, \mathcal{O}, T)$ of the form $e^{C(\Omega, \mathcal{O})(1+1 / T)}$.

Step 3 . From the equation verified by $\varphi$, we readily obtain

$$
\|\varphi(0)\|_{L^{2}(\Omega)}^{2} \leq \frac{2}{T} \int_{T / 4}^{3 T / 4}\|\varphi(t)\|_{L^{2}(\Omega)}^{2} d t
$$

This, together with (1.22), gives the observability inequality (1.3).

In view of Theorem 1.1, we have the following.

THEOREM 1.4. The system (1.1) is null controllable with controls $v \in L^{2}(\mathcal{O} \times$ $(0, T))$ that verify

$$
\|v\|_{L^{2}(\mathcal{O} \times(0, T))} \leq C\left\|y^{0}\right\|_{L^{2}(\Omega)},
$$

where the constant $C$ is of the form $e^{C(\Omega, \mathcal{O})(1+1 / T)}$.

Let us now give the proof of the Carleman estimate (1.18). The proof we present here is based on the ideas in [30]. We will try to keep an explicit dependence of all the constants with respect to the parameters $s$ and $\lambda$ and the final time $T$. This follows the spirit of [27] and will be crucial for the analysis of similar nonlinear problems.

Proof of Lemma 1.3. For simplicity, we will divide the proof into three steps.

Step 1. Change of variables and plan of what follows. In this step, we set the differential equation satisfied by a new function $\psi$, which will be $q$ up to a weight function.

Thus, let us introduce the new functions $\psi=e^{-s \alpha} q$ and $g=e^{-s \alpha} f$, where we have denoted $f=q_{t}+\Delta q$. Then we easily obtain that

$$
M_{1} \psi+M_{2} \psi=g_{s, \lambda},
$$

where

$$
\begin{aligned}
& M_{1} \psi=-2 s \lambda^{2}\left|\nabla \eta^{0}\right|^{2} \xi \psi-2 s \lambda \xi \nabla \eta^{0} \cdot \nabla \psi+\psi_{t}, \\
& M_{2} \psi=s^{2} \lambda^{2}\left|\nabla \eta^{0}\right|^{2} \xi^{2} \psi+\Delta \psi+s \alpha_{t} \psi,
\end{aligned}
$$

and

$$
g_{s, \lambda}=g+s \lambda \Delta \eta^{0} \xi \psi-s \lambda^{2}\left|\nabla \eta^{0}\right|^{2} \xi \psi .
$$

To simplify the notation, we will denote by $\left(M_{i} \psi\right)_{j}(1 \leq i \leq 2,1 \leq j \leq 3)$ the $j$ th term in the expression of $M_{i} \psi$ given in (1.26).

Observe that, at first sight, it seems "natural" to put $\left(M_{1} \psi\right)_{1}$ on the right-hand side of (1.25). However, we have decided to keep it on the left, because it will serve to produce a positive term on $|\nabla \psi|^{2}$ (see the scalar product $\left(\left(M_{1} \psi\right)_{1},\left(M_{2} \psi\right)_{2}\right)_{L^{2}(Q)}$ below).

With the previous notation, we have from (1.25)

$$
\left\|M_{1} \psi\right\|_{L^{2}(Q)}^{2}+\left\|M_{2} \psi\right\|_{L^{2}(Q)}^{2}+2 \sum_{i, j=1}^{3}\left(\left(M_{1} \psi\right)_{i},\left(M_{2} \psi\right)_{j}\right)_{L^{2}(Q)}=\left\|g_{s, \lambda}\right\|_{L^{2}(Q)}^{2} .
$$

In the following steps, we will see that the definition we have made of $\alpha$ makes $2\left(M_{1} \psi, M_{2} \psi\right)_{L^{2}(Q)}$ positive up to several terms that can be controlled whenever we make an appropriate choice of the parameters $s$ and $\lambda$.

More precisely, in the second step we will make the computations of the double products $2\left(M_{1} \psi, M_{2} \psi\right)_{L^{2}(Q)}$. This will give an inequality with two global terms of 
$|\psi|^{2}$ and $|\nabla \psi|^{2}$ on the left-hand side, while two local terms of $|\psi|^{2}$ and $|\nabla \psi|^{2}$ will appear on the right-hand side (see (1.57)). In the third step we will add two terms (involving $\psi_{t}$ and $\Delta \psi$ ) to the left of (1.57). This will help us to eliminate the local term containing $\nabla \psi$ that appears on the right-hand side and will provide a Carleman inequality for the function $\psi$ (see (1.63)). Finally, we will turn back to the original function $q$ and deduce the inequality (1.18).

Step 2. First estimates. In this step, we will develop the nine terms appearing in $\left(M_{1} \psi, M_{2} \psi\right)_{L^{2}(Q)}$. For this, we will integrate by parts several times with respect to the space and time variables, so derivatives of the weight functions will be involved. Actually, we will use the estimates

$$
\begin{aligned}
\partial_{i} \alpha & =-\partial_{i} \xi=-\lambda \partial_{i} \eta^{0} \xi \leq C \lambda \xi, \\
\alpha_{t} & =-(T-2 t) \frac{e^{2 \lambda m\left\|\eta^{0}\right\|_{\infty}-e^{\lambda\left(m\left\|\eta^{0}\right\|_{\infty}+\eta^{0}\right)}}}{t^{2}(T-t)^{2}} \leq C T \xi^{2},
\end{aligned}
$$

where $C$ is a positive constant depending only on $\Omega$ and $\mathcal{O}$.

The last inequality follows from the fact that

$$
e^{2 \lambda m\left\|\eta^{0}\right\|_{\infty}} \leq e^{2 \lambda\left(m\left\|\eta^{0}\right\|_{\infty}+\eta^{0}\right)} \quad \text { in } \Omega .
$$

First, we have

$$
\left(\left(M_{1} \psi\right)_{1},\left(M_{2} \psi\right)_{1}\right)_{L^{2}(Q)}=-2 s^{3} \lambda^{4} \iint_{Q}\left|\nabla \eta^{0}\right|^{4} \xi^{3}|\psi|^{2} d x d t=A .
$$

Then

$$
\begin{aligned}
\left(\left(M_{1} \psi\right)_{2},\left(M_{2} \psi\right)_{1}\right)_{L^{2}(Q)}= & -2 s^{3} \lambda^{3} \iint_{Q}\left|\nabla \eta^{0}\right|^{2} \xi^{3}\left(\nabla \eta^{0} \cdot \nabla \psi\right) \psi d x d t \\
= & 3 s^{3} \lambda^{4} \iint_{Q}\left|\nabla \eta^{0}\right|^{4} \xi^{3}|\psi|^{2} d x d t \\
& +s^{3} \lambda^{3} \iint_{Q} \Delta \eta^{0}\left|\nabla \eta^{0}\right|^{2} \xi^{3}|\psi|^{2} d x d t \\
& +2 s^{3} \lambda^{3} \sum_{i, j=1}^{N} \iint_{Q} \partial_{i} \eta^{0} \partial_{i j} \eta^{0} \partial_{j} \eta^{0} \xi^{3}|\psi|^{2} d x d t \\
= & B_{1}+B_{2}+B_{3} .
\end{aligned}
$$

We clearly have that $A+B_{1}$ is a positive term. As a consequence of the properties of $\eta^{0}$ (see Lemma 1.2), we have

$$
\begin{aligned}
A+B_{1}= & s^{3} \lambda^{4} \iint_{Q}\left|\nabla \eta^{0}\right|^{4} \xi^{3}|\psi|^{2} d x d t \geq C s^{3} \lambda^{4} \iint_{Q} \xi^{3}|\psi|^{2} d x d t \\
& -C s^{3} \lambda^{4} \iint_{\omega \times(0, T)} \xi^{3}|\psi|^{2} d x d t=\widetilde{A}-\widetilde{B}
\end{aligned}
$$

for some $C=C(\Omega, \mathcal{O})$. The first of these last two integrals $(\widetilde{A})$ will stay on the left-hand side, while the second one $(\widetilde{B})$ will go to the right-hand side.

The terms $B_{2}$ and $B_{3}$ are absorbed by $\widetilde{A}$ by simply taking $\lambda \geq C$, since $\eta^{0} \in$ $C^{2}(\bar{\Omega})$. 
We also have

$$
\begin{aligned}
\left(\left(M_{1} \psi\right)_{3},\left(M_{2} \psi\right)_{1}\right)_{L^{2}(Q)} & =s^{2} \lambda^{2} \iint_{Q}\left|\nabla \eta^{0}\right|^{2} \xi^{2} \psi_{t} \psi d x d t \\
& =-s^{2} \lambda^{2} \iint_{Q}\left|\nabla \eta^{0}\right|^{2} \xi \xi_{t}|\psi|^{2} d x d t,
\end{aligned}
$$

which, by virtue of (1.29), is bounded by

$$
C s^{2} \lambda^{2} T \iint_{Q} \xi^{3}|\psi|^{2} d x d t
$$

This term can also be absorbed if we take $\lambda \geq 1$ and $s \geq C(\Omega, \mathcal{O}) T$.

Consequently, we have

$$
\begin{aligned}
\left(M_{1} \psi,\left(M_{2} \psi\right)_{1}\right)_{L^{2}(Q)}= & \left(\left(M_{1} \psi\right)_{1}+\left(M_{1} \psi\right)_{2}+\left(M_{1} \psi\right)_{3},\left(M_{2} \psi\right)_{1}\right)_{L^{2}(Q)} \\
\geq & C s^{3} \lambda^{4} \iint_{Q} \xi^{3}|\psi|^{2} d x d t \\
& -C s^{3} \lambda^{4} \iint_{\omega \times(0, T)} \xi^{3}|\psi|^{2} d x d t
\end{aligned}
$$

for any $\lambda \geq C(\Omega, \mathcal{O})$ and $s \geq C(\Omega, \mathcal{O}) T$.

On the other hand, we have

$$
\begin{aligned}
\left(\left(M_{1} \psi\right)_{1},\left(M_{2} \psi\right)_{2}\right)_{L^{2}(Q)}= & -2 s \lambda^{2} \iint_{Q}\left|\nabla \eta^{0}\right|^{2} \xi \Delta \psi \psi d x d t \\
= & 2 s \lambda^{2} \iint_{Q}\left|\nabla \eta^{0}\right|^{2} \xi|\nabla \psi|^{2} d x d t \\
& +4 s \lambda^{2} \sum_{i, j=1}^{N} \iint_{Q} \partial_{i} \eta^{0} \partial_{i j} \eta^{0} \xi \partial_{j} \psi \psi d x d t \\
& +2 s \lambda^{3} \iint_{Q}\left|\nabla \eta^{0}\right|^{2} \xi\left(\nabla \eta^{0} \cdot \nabla \psi\right) \psi d x d t \\
= & C_{1}+C_{2}+C_{3} .
\end{aligned}
$$

We will keep $C_{1}$ on the left-hand side. For $C_{2}$ and $C_{3}$, we have

$$
C_{2} \leq C s \lambda^{4} \iint_{Q} \xi|\psi|^{2} d x d t+C s \iint_{Q} \xi|\nabla \psi|^{2} d x d t
$$

and

$$
C_{3} \leq C s^{2} \lambda^{4} \iint_{Q} \xi^{2}|\psi|^{2} d x d t+C \lambda^{2} \iint_{Q}|\nabla \psi|^{2} d x d t .
$$

Therefore, taking $s \geq C T^{2}$, we find that

$$
\begin{aligned}
C_{1}+C_{2}+C_{3} \geq & 2 s \lambda^{2} \iint_{Q}\left|\nabla \eta^{0}\right|^{2} \xi|\nabla \psi|^{2} d x d t \\
& -C s^{2} \lambda^{4} \iint_{Q} \xi^{2}|\psi|^{2} d x d t-C \iint_{Q}\left(s \xi+\lambda^{2}\right)|\nabla \psi|^{2} d x d t .
\end{aligned}
$$


We also have

$$
\begin{aligned}
\left(\left(M_{1} \psi\right)_{2},\left(M_{2} \psi\right)_{2}\right)_{L^{2}(Q)}= & -2 s \lambda \iint_{Q} \xi\left(\nabla \eta^{0} \cdot \nabla \psi\right) \Delta \psi d x d t \\
= & -2 s \lambda \iint_{\Sigma} \frac{\partial \eta^{0}}{\partial n} \xi\left|\frac{\partial \psi}{\partial n}\right|^{2} d \sigma d t \\
& +2 s \lambda \sum_{i, j=1}^{N} \iint_{Q} \partial_{i j} \eta^{0} \xi \partial_{i} \psi \partial_{j} \psi d x d t \\
& +2 s \lambda^{2} \iint_{Q} \xi\left|\nabla \eta^{0} \cdot \nabla \psi\right|^{2} d x d t \\
& +s \lambda \iint_{Q} \xi \nabla \eta^{0} \cdot \nabla|\nabla \psi|^{2} d x d t \\
= & D_{1}+D_{2}+D_{3}+D_{4} .
\end{aligned}
$$

Let us remark that $D_{3}$ is a positive term. Furthermore,

$$
D_{2} \leq C s \lambda \iint_{Q} \xi|\nabla \psi|^{2} d x d t .
$$

After some additional computations we also see that

$$
\begin{aligned}
D_{4}= & s \lambda \iint_{Q} \xi \nabla \eta^{0} \cdot \nabla|\nabla \psi|^{2} d x d t=s \lambda \iint_{\Sigma} \xi \frac{\partial \eta^{0}}{\partial n}\left|\frac{\partial \psi}{\partial n}\right|^{2} d \sigma d t \\
& -s \lambda^{2} \iint_{Q}\left|\nabla \eta^{0}\right|^{2} \xi|\nabla \psi|^{2} d x d t-s \lambda \iint_{Q} \Delta \eta^{0} \xi|\nabla \psi|^{2} d x d t \\
= & D_{41}+D_{42}+D_{43} .
\end{aligned}
$$

By virtue of the properties satisfied by $\eta^{0}$, we notice that $D_{1}+D_{41} \geq 0$ and that $D_{43}$ can be bounded in the same way as $D_{2}$.

Consequently,

$$
\begin{aligned}
D_{1}+D_{2}+D_{3}+D_{4} \geq & -s \lambda^{2} \iint_{Q}\left|\nabla \eta^{0}\right|^{2} \xi|\nabla \psi|^{2} d x d t \\
& -C s \lambda \iint_{Q} \xi|\nabla \psi|^{2} d x d t
\end{aligned}
$$

Additionally, we find that

$$
\left(\left(M_{1} \psi\right)_{3},\left(M_{2} \psi\right)_{2}\right)_{L^{2}(Q)}=\iint_{Q} \psi_{t} \Delta \psi d x d t=0 .
$$

From (1.40)-(1.45), we deduce that

$$
\begin{aligned}
\left(M_{1} \psi,\left(M_{2} \psi\right)_{2}\right)_{L^{2}(Q)}= & \left(\left(M_{1} \psi\right)_{1}+\left(M_{1} \psi\right)_{2}+\left(M_{1} \psi\right)_{3},\left(M_{2} \psi\right)_{2}\right)_{L^{2}(Q)} \\
\geq & s \lambda^{2} \iint_{Q}\left|\nabla \eta^{0}\right|^{2} \xi|\nabla \psi|^{2} d x d t \\
& -C s^{2} \lambda^{4} \iint_{Q} \xi^{2}|\psi|^{2} d x d t \\
& -C \iint_{Q}\left(s \lambda \xi+\lambda^{2}\right)|\nabla \psi|^{2} d x d t
\end{aligned}
$$

for $\lambda \geq 1$ and $s \geq C T^{2}$. 
Hence, we have the following for $\lambda \geq C(\Omega, \mathcal{O})$ and $s \geq C(\Omega, \mathcal{O}) T^{2}$ :

$$
\begin{aligned}
\left(M_{1} \psi,\left(M_{2} \psi\right)_{2}\right)_{L^{2}(Q)} \geq & C s \lambda^{2} \iint_{Q} \xi|\nabla \psi|^{2} d x d t \\
& -C s^{2} \lambda^{4} \iint_{Q} \xi^{2}|\psi|^{2} d x d t \\
& -C s \lambda^{2} \iint_{\omega \times(0, T)} \xi|\nabla \psi|^{2} d x d t .
\end{aligned}
$$

Let us now consider the scalar product

$$
\begin{aligned}
\left(\left(M_{1} \psi\right)_{1},\left(M_{2} \psi\right)_{3}\right)_{L^{2}(Q)} & =-2 s^{2} \lambda^{2} \iint_{Q}\left|\nabla \eta^{0}\right|^{2} \alpha_{t} \xi|\psi|^{2} d x d t \\
& \leq C s^{2} \lambda^{2} T \iint_{Q} \xi^{3}|\psi|^{2} d x d t .
\end{aligned}
$$

Obviously, this is absorbed by $\widetilde{A}$ if we take $\lambda \geq 1$ and $s \geq C T$.

Furthermore,

$$
\begin{aligned}
\left(\left(M_{1} \psi\right)_{2},\left(M_{2} \psi\right)_{3}\right)_{L^{2}(Q)}= & -2 s^{2} \lambda \iint_{Q} \alpha_{t} \xi\left(\nabla \eta^{0} \cdot \nabla \psi\right) \psi d x d t \\
= & s^{2} \lambda^{2} \iint_{Q} \alpha_{t}\left|\nabla \eta^{0}\right|^{2} \xi|\psi|^{2} d x d t \\
& +s^{2} \lambda \iint_{Q} \nabla \alpha_{t} \cdot \nabla \eta^{0} \xi|\psi|^{2} d x d t \\
& +s^{2} \lambda \iint_{Q} \alpha_{t} \Delta \eta^{0} \xi|\psi|^{2} d x d t
\end{aligned}
$$

From (1.29), one can easily check that the previous three terms can be bounded (if $\lambda \geq 1$ ) by

$$
C s^{2} \lambda^{2} T \iint_{Q} \xi^{3}|\psi|^{2} d x d t
$$

Thus, we have

$$
\left(\left(M_{1} \psi\right)_{2},\left(M_{2} \psi\right)_{3}\right)_{L^{2}(Q)} \geq-C s^{2} \lambda^{2} T \iint_{Q} \xi^{3}|\psi|^{2} d x d t .
$$

Finally, we have

$$
\begin{aligned}
\left(\left(M_{1} \psi\right)_{3},\left(M_{2} \psi\right)_{3}\right)_{L^{2}(Q)} & =s \iint_{Q} \alpha_{t} \psi_{t} \psi d x d t \\
& =-\frac{1}{2} s \iint_{Q} \alpha_{t t}|\psi|^{2} d x d t \leq C s T^{2} \iint_{Q} \xi^{3}|\psi|^{2} d x d t
\end{aligned}
$$

since

$$
\alpha_{t t} \leq C \xi^{2}\left(1+T^{2} \xi\right) \leq C T^{2} \xi^{3} .
$$


From (1.48)-(1.52), we deduce for $\lambda \geq C(\Omega, \mathcal{O})$ and $s \geq C(\Omega, \mathcal{O}) T$ that

$$
\begin{aligned}
\left(M_{1} \psi,\left(M_{2} \psi\right)_{3}\right)_{L^{2}(Q)} & =\left(\left(M_{1} \psi\right)_{1}+\left(M_{1} \psi\right)_{2}+\left(M_{1} \psi\right)_{3},\left(M_{2} \psi\right)_{3}\right)_{L^{2}(Q)} \\
& \geq-C s^{3} \lambda^{2} \iint_{Q} \xi^{3}|\psi|^{2} d x d t
\end{aligned}
$$

Taking into account (1.36), (1.47), and (1.54), we obtain

$$
\begin{aligned}
\left(M_{1} \psi, M_{2} \psi\right)_{L^{2}(Q)} \geq & C \iint_{Q}\left(s \lambda^{2} \xi|\nabla \psi|^{2}+s^{3} \lambda^{4} \xi^{3}|\psi|^{2}\right) d x d t \\
& -C \iint_{\omega \times(0, T)}\left(s \lambda^{2} \xi|\nabla \psi|^{2}+s^{3} \lambda^{4} \xi^{3}|\psi|^{2}\right) d x d t
\end{aligned}
$$

for any $\lambda \geq C(\Omega, \mathcal{O})$ and $s \geq C(\Omega, \mathcal{O})\left(T+T^{2}\right)$. Using (1.28), this gives

$$
\begin{aligned}
& \left\|M_{1} \psi\right\|_{L^{2}(Q)}^{2}+\left\|M_{2} \psi\right\|_{L^{2}(Q)}^{2}+\iint_{Q}\left(s \lambda^{2} \xi|\nabla \psi|^{2}+s^{3} \lambda^{4} \xi^{3}|\psi|^{2}\right) d x d t \\
& \leq C\left(\left\|g_{s, \lambda}\right\|_{L^{2}(Q)}^{2}+\iint_{\omega \times(0, T)}\left(s \lambda^{2} \xi|\nabla \psi|^{2}+s^{3} \lambda^{4} \xi^{3}|\psi|^{2}\right) d x d t\right) \\
& \leq C\left(\iint_{Q} e^{-2 s \alpha}|f|^{2} d x d t+s^{2} \lambda^{4} \iint_{Q} \xi^{2}|\psi|^{2} d x d t\right. \\
& \left.\quad+s \lambda^{2} \iint_{\omega \times(0, T)} \xi|\nabla \psi|^{2} d x d t+s^{3} \lambda^{4} \iint_{\omega \times(0, T)} \xi^{3}|\psi|^{2} d x d t\right) .
\end{aligned}
$$

Thus, we also have

$$
\begin{gathered}
\left\|M_{1} \psi\right\|_{L^{2}(Q)}^{2}+\left\|M_{2} \psi\right\|_{L^{2}(Q)}^{2}+\iint_{Q}\left(s \lambda^{2} \xi|\nabla \psi|^{2}+s^{3} \lambda^{4} \xi^{3}|\psi|^{2}\right) d x d t \\
\leq C\left(\iint_{Q} e^{-2 s \alpha}|f|^{2} d x d t+s \lambda^{2} \iint_{\omega \times(0, T)} \xi|\nabla \psi|^{2} d x d t\right. \\
\left.\quad+s^{3} \lambda^{4} \iint_{\omega \times(0, T)} \xi^{3}|\psi|^{2} d x d t\right)
\end{gathered}
$$

for $\lambda \geq C(\Omega, \mathcal{O})$ and $s \geq C(\Omega, \mathcal{O})\left(T+T^{2}\right)$.

Step 3 . Indirect estimates and conclusion. The final step will be to add integrals of $|\Delta \psi|^{2}$ and $\left|\psi_{t}\right|^{2}$ to the left-hand side of (1.57). This can be made using the expressions of $M_{i} \psi(i=1,2)$. Indeed, from (1.26) we have

$$
\begin{aligned}
s^{-1} \iint_{Q} \xi^{-1}\left|\psi_{t}\right|^{2} d x d t \leq & C\left(s \lambda^{2} \iint_{Q} \xi|\nabla \psi|^{2} d x d t\right. \\
& \left.+s \lambda^{4} \iint_{Q} \xi|\psi|^{2} d x d t+\left\|M_{1} \psi\right\|_{L^{2}(Q)}^{2}\right)
\end{aligned}
$$

and

$$
\begin{aligned}
s^{-1} \iint_{Q} \xi^{-1}|\Delta \psi|^{2} d x d t \leq & C\left(s^{3} \lambda^{4} \iint_{Q} \xi^{3}|\psi|^{2} d x d t\right. \\
& \left.+s T^{2} \iint_{Q} \xi^{3}|\psi|^{2} d x d t+\left\|M_{2} \psi\right\|_{L^{2}(Q)}^{2}\right)
\end{aligned}
$$


for $s \geq C T^{2}$. Accordingly, we deduce from (1.57) that

$$
\begin{gathered}
\iint_{Q}\left(s^{-1} \xi^{-1}\left(\left|\psi_{t}\right|^{2}+|\Delta \psi|^{2}\right)+s \lambda^{2} \xi|\nabla \psi|^{2}+s^{3} \lambda^{4} \xi^{3}|\psi|^{2}\right) d x d t \\
\leq C\left(\iint_{Q} e^{-2 s \alpha}|f|^{2} d x d t+s \lambda^{2} \iint_{\omega \times(0, T)} \xi|\nabla \psi|^{2} d x d t\right. \\
\left.\quad+s^{3} \lambda^{4} \iint_{\omega \times(0, T)} \xi^{3}|\psi|^{2} d x d t\right)
\end{gathered}
$$

for any $\lambda \geq C(\Omega, \mathcal{O})$ and $s \geq C(\Omega, \mathcal{O})\left(T+T^{2}\right)$.

We are now ready to eliminate the second integral on the right-hand side. To this end, let us introduce a function $\theta=\theta(x)$, with

$$
\theta \in C_{c}^{2}(\mathcal{O}), \quad \theta \equiv 1 \quad \text { in } \quad \omega, \quad 0 \leq \theta \leq 1
$$

and let us make some computations. We have

$$
\begin{aligned}
s \lambda^{2} & \iint_{\omega \times(0, T)} \xi|\nabla \psi|^{2} d x d t \leq s \lambda^{2} \iint_{\mathcal{O} \times(0, T)} \theta \xi|\nabla \psi|^{2} d x d t \\
= & -s \lambda^{2} \iint_{\mathcal{O} \times(0, T)} \theta \xi \Delta \psi \psi d x d t \\
& -s \lambda^{2} \iint_{\mathcal{O} \times(0, T)} \xi(\nabla \theta \cdot \nabla \psi) \psi d x d t \\
& -s \lambda^{3} \iint_{\mathcal{O} \times(0, T)} \theta \xi\left(\nabla \eta^{0} \cdot \nabla \psi\right) \psi d x d t \\
\leq & \varepsilon s^{-1} \iint_{\mathcal{O} \times(0, T)} \xi^{-1}|\Delta \psi|^{2} d x d t \\
& +C\left(s^{3} \lambda^{4} \iint_{\mathcal{O} \times(0, T)} \xi^{3}|\psi|^{2} d x d t+s \lambda^{4} \iint_{\mathcal{O} \times(0, T)} \xi|\psi|^{2} d x d t\right)
\end{aligned}
$$

for a small enough constant $\varepsilon=\varepsilon(\Omega, \mathcal{O})>0$ and where we have used the fact that $\lambda \geq 1$. Hence, we can eliminate the integral of $|\nabla \psi|^{2}$ on the right-hand side of (1.60), paying the price of having $|\psi|^{2}$ in $\mathcal{O} \times(0, T)$. From (1.60) and this remark, we deduce that

$$
\begin{aligned}
& \iint_{Q}\left(s^{-1} \xi^{-1}\left(\left|\psi_{t}\right|^{2}+|\Delta \psi|^{2}\right)+s \lambda^{2} \xi|\nabla \psi|^{2}+s^{3} \lambda^{4} \xi^{3}|\psi|^{2}\right) d x d t \\
& \quad \leq C\left(\iint_{Q} e^{-2 s \alpha}|f|^{2} d x d t+s^{3} \lambda^{4} \iint_{\mathcal{O} \times(0, T)} \xi^{3}|\psi|^{2} d x d t\right)
\end{aligned}
$$

for $\lambda \geq C(\Omega, \mathcal{O})$ and $s \geq C(\Omega, \mathcal{O})\left(T+T^{2}\right)$.

We finally turn back to our original function, which was given by $q=e^{s \alpha} \psi$. For 
the moment, we have

$$
\begin{aligned}
& s^{-1} \iint_{Q} \xi^{-1}\left|\psi_{t}\right|^{2} d x d t+s^{-1} \iint_{Q} \xi^{-1}|\Delta \psi|^{2} d x d t \\
& \quad+s \lambda^{2} \iint_{Q} \xi|\nabla \psi|^{2} d x d t+s^{3} \lambda^{4} \iint_{Q} e^{-2 s \alpha} \xi^{3}|q|^{2} d x d t \\
& \quad \leq C\left(\iint_{Q} e^{-2 s \alpha}|f|^{2} d x d t+s^{3} \lambda^{4} \iint_{\mathcal{O} \times(0, T)} e^{-2 s \alpha} \xi^{3}|q|^{2} d x d t\right) .
\end{aligned}
$$

Using that

$$
\nabla q=e^{s \alpha}\left(\nabla \psi-s \lambda \nabla \eta^{0} \xi \psi\right)
$$

we find

$$
\begin{aligned}
s \lambda^{2} \iint_{Q} e^{-2 s \alpha} \xi|\nabla q|^{2} d x d t \leq & C s \lambda^{2} \iint_{Q} \xi|\nabla \psi|^{2} d x d t \\
& +C s^{3} \lambda^{4} \iint_{Q} e^{-2 s \alpha} \xi^{3}|q|^{2} d x d t .
\end{aligned}
$$

Consequently, we can add the previous integral of $|\nabla q|^{2}$ to the left-hand side of (1.64):

$$
\begin{aligned}
& s^{-1} \iint_{Q} \xi^{-1}\left|\psi_{t}\right|^{2} d x d t+s^{-1} \iint_{Q} \xi^{-1}|\Delta \psi|^{2} d x d t \\
& \quad+s \lambda^{2} \iint_{Q} \xi|\nabla q|^{2} d x d t+s^{3} \lambda^{4} \iint_{Q} e^{-2 s \alpha} \xi^{3}|q|^{2} d x d t \\
& \quad \leq C\left(\iint_{Q} e^{-2 s \alpha}|f|^{2} d x d t+s^{3} \lambda^{4} \iint_{\mathcal{O} \times(0, T)} e^{-2 s \alpha} \xi^{3}|q|^{2} d x d t\right) .
\end{aligned}
$$

For $\Delta q$, we use the identity

$$
\Delta \psi=e^{-s \alpha}\left(\Delta q+s \lambda \Delta \eta^{0} \xi q+s \lambda^{2}\left|\nabla \eta^{0}\right|^{2} \xi q+2 s \lambda \xi \nabla \eta^{0} \cdot \nabla q+s^{2} \lambda^{2}\left|\nabla \eta^{0}\right|^{2} \xi^{2} q\right)
$$

and obtain

$$
\begin{aligned}
& s^{-1} \iint_{Q} e^{-2 s \alpha} \xi^{-1}|\Delta q|^{2} d x d t \leq C\left(s^{-1} \iint_{Q} \xi^{-1}|\Delta \psi|^{2} d x d t\right. \\
& \quad+s \lambda^{2} \iint_{Q} e^{-2 s \alpha} \xi|q|^{2} d x d t+s \lambda^{4} \iint_{Q} e^{-2 s \alpha} \xi|q|^{2} d x d t \\
& \left.\quad+s \lambda^{2} \iint_{Q} e^{-2 s \alpha} \xi|\nabla q|^{2} d x d t+s^{3} \lambda^{4} \iint_{Q} e^{-2 s \alpha} \xi^{3}|q|^{2} d x d t\right) .
\end{aligned}
$$

Finally, for $q_{t}$ we get

$$
\begin{aligned}
s^{-1} \iint_{Q} e^{-2 s \alpha} \xi^{-1}\left|q_{t}\right|^{2} d x d t \leq & C\left(s^{-1} \iint_{Q} \xi^{-1}\left|\psi_{t}\right|^{2} d x d t\right. \\
& \left.+s T^{2} \iint_{Q} e^{-2 s \alpha} \xi^{3}|q|^{2} d x d t\right),
\end{aligned}
$$


where we have used the identity $q_{t}=e^{s \alpha}\left(\psi_{t}+s \alpha_{t} \psi\right)$. Thus, taking $\lambda \geq 1$ and $s \geq C(\Omega, \mathcal{O})\left(T+T^{2}\right)$, we are able to introduce all the terms involving $|\Delta q|^{2}$ and $\left|q_{t}\right|^{2}$ on the left-hand side of (1.64). This gives (1.18) and concludes the proof of Lemma 1.3 .

Remark 4. It would be very interesting to know whether the powers of $s$ and $\lambda$ arising in (1.18) are optimal. In other words, would it be possible to deduce an inequality like (1.18) with higher powers of $s$ and $\lambda$ accompanying the terms on the left-hand side? A positive answer to this would lead to the controllability of nonlinear parabolic systems more general than those considered in subsections 1.3.2 and 2.2.1.

1.3. Applications and generalizations. There are many other similar systems for which null controllability properties can be analyzed as before. Let us mention some of them.

1.3.1. General parabolic linear systems. We will now try to apply the Carleman inequality obtained in the previous subsection to systems where the heat equation has a zero order term and a first order term in the divergence form. More precisely, we would like to prove the null controllability of the system

$$
\begin{cases}y_{t}-\Delta y+\nabla \cdot(y B(x, t))+a(x, t) y=v 1_{\mathcal{O}} & \text { in } Q, \\ y=0 & \text { on } \Sigma, \\ y(0)=y^{0} & \text { in } \Omega,\end{cases}
$$

where $y^{0} \in L^{2}(\Omega), a \in L^{\infty}(Q)$, and $B \in L^{\infty}(Q)^{N}$.

In this case, the associated adjoint system is the following:

$$
\begin{cases}-\varphi_{t}-\Delta \varphi-B(x, t) \cdot \nabla \varphi+a(x, t) \varphi=0 & \text { in } Q, \\ \varphi=0 & \text { on } \Sigma, \\ \varphi(T)=\varphi^{0} & \text { in } \Omega .\end{cases}
$$

A result similar to Theorem 1.1 holds for systems (1.71) and (1.72). Consequently, what we have to do is prove an observability inequality for the solutions to (1.72).

In this situation, Lemma 1.3 provides a first estimate of the kind (1.18) (with $q$ replaced by $\varphi$ ), with two additional terms on the right-hand side, namely,

$$
C \iint_{Q} e^{-2 s \alpha}|a \varphi|^{2} d x d t \text { and } C \iint_{Q} e^{-2 s \alpha}|B \cdot \nabla \varphi|^{2} d x d t .
$$

But these can be absorbed by the left-hand side if we take $s$ large enough. Indeed, it suffices to take

$$
s \geq C(\Omega, \mathcal{O}) T^{2}\left(\|a\|_{\infty}^{2 / 3}+\|B\|_{\infty}^{2}\right)
$$

to have

$$
\begin{aligned}
& C\left(\iint_{Q} e^{-2 s \alpha}|a \varphi|^{2} d x d t+\iint_{Q} e^{-2 s \alpha}|B \cdot \nabla \varphi|^{2} d x d t\right) \\
& \quad \leq \frac{1}{2} s^{3} \lambda^{4} \iint_{Q} e^{-2 s \alpha} \xi^{3}|\varphi|^{2} d x d t+\frac{1}{2} s \lambda^{2} \iint_{Q} e^{-2 s \alpha} \xi|\nabla \varphi|^{2} d x d t .
\end{aligned}
$$

Arguing as we did when we proved the observability inequality for the solutions to (1.2), we easily find

$$
\iint_{\Omega \times(T / 4,3 T / 4)}|\varphi|^{2} d x d t \leq C(\Omega, \mathcal{O}, T, a, B) \iint_{\mathcal{O} \times(0, T)}|\varphi|^{2} d x d t
$$


with a constant

$$
C(\Omega, \mathcal{O}, T, a, B)=\exp \left\{C\left(1+1 / T+\|a\|_{\infty}^{2 / 3}+\|B\|_{\infty}^{2}\right)\right\} .
$$

On the other hand, the dissipativity of $\varphi$ yields

$$
\|\varphi(0)\|_{L^{2}(\Omega)}^{2} \leq \exp \left\{C T\left(\|a\|_{\infty}+\|B\|_{\infty}^{2}\right)\right\}\|\varphi(t)\|_{L^{2}(\Omega)}^{2}
$$

for all $t \in(T / 4,3 T / 4)$. Combining (1.76) and (1.78), we see that

$$
\|\varphi(0)\|_{L^{2}(\Omega)}^{2} \leq e^{C\left(1+1 / T+\|a\|_{\infty}^{2 / 3}+T\|a\|_{\infty}+(1+T)\|B\|_{\infty}^{2}\right)} \iint_{\mathcal{O} \times(0, T)}|\varphi|^{2} d x d t
$$

for all the solutions of (1.72) associated to final data $\varphi^{0} \in L^{2}(\Omega)$. As mentioned above (see Theorem 1.1), this implies the null controllability of (1.71).

THEOREM 1.5. System (1.71) is null controllable, with controls $v$ satisfying

$$
\|v\|_{L^{2}(\mathcal{O} \times(0, T))} \leq C(\Omega, \mathcal{O}, T, a, B)\left\|y^{0}\right\|_{L^{2}(\Omega)},
$$

where

$$
C(\Omega, \mathcal{O}, T, a, B)=e^{C(\Omega, \mathcal{O})\left(1+1 / T+\|a\|_{\infty}^{2 / 3}+T\|a\|_{\infty}+(1+T)\|B\|_{\infty}^{2}\right)} .
$$

Remark 5. In a recent paper, Seidman [54] looked at the qualitative asymptotic behavior of the constants found in estimates of the kind (1.80) with respect to relevant parameters. In particular, the blow-up of $C(\Omega, \mathcal{O}, T, a, B)$ as $T \rightarrow 0$ has been analyzed in some particular cases.

The next step will be to establish the null controllability for a general linear heat equation system with first order terms and coefficients in $L^{\infty}(Q)$, i.e., an equation of the form

$$
y_{t}-\Delta y+B(x, t) \cdot \nabla y+a(x, t) y=v 1_{\mathcal{O}},
$$

with $a \in L^{\infty}(Q)$ and $B \in L^{\infty}(Q)^{N}$. A different Carleman inequality is now required since, in the corresponding adjoint equation, there is the term $-\nabla \cdot(\varphi B(x, t))$, which belongs to $L^{2}\left(0, T ; H^{-1}(\Omega)\right)$. This will be treated in section 2 using arguments from [39].

1.3.2. Extension to some semilinear systems. We will now consider possible extensions of the null controllability results above to nonlinear problems of the form

$$
\begin{cases}y_{t}-\Delta y+f(y)=v 1_{\mathcal{O}} & \text { in } Q, \\ y=0 & \text { on } \Sigma, \\ y(0)=y^{0} & \text { in } \Omega,\end{cases}
$$

where

$$
f \in C^{1}(\mathbf{R}) \text { (for instance), } \quad f(0)=0, \quad \text { and } \quad|f(s)| \leq C(1+|s|) .
$$

The classical strategy used in [36] and [30] relies on the introduction of a mapping

$$
z \in L^{2}(Q) \mapsto y_{z} \in L^{2}(Q),
$$


where $y_{z}$ is, together with $v_{z}$, a solution of

$$
\begin{cases}\left(y_{z}\right)_{t}-\Delta y_{z}+g(z) y_{z}=v_{z} 1_{\mathcal{O}} & \text { in } Q \\ y_{z}=0 & \text { on } \Sigma \\ y_{z}(0)=y^{0} & \text { in } \Omega\end{cases}
$$

such that

$$
y_{z}(T)=0
$$

and (1.80) holds for $v_{z}$ with $a=g(z)$ and $B \equiv 0$. Here, $g$ is given by

$$
g(s)= \begin{cases}\frac{f(s)}{s} & \text { if } \quad s \neq 0 \\ f^{\prime}(0) & \text { if } \quad s=0 .\end{cases}
$$

Using the previous results and arguing as in [36], it can be seen that this mapping can be correctly defined. It can also be proved that it is continuous and compact. Furthermore, in view of (1.84), the estimate (1.80) written for $v_{z}$, and the classical estimates for $y_{z}$, it maps the whole space $L^{2}(Q)$ into a ball. Hence, from Schauder's theorem, we deduce that this mapping possesses a fixed point and, consequently, the following holds.

THEOREM 1.6. Under conditions (1.84) on $f$, system (1.83) is null controllable, with controls $v$ satisfying

$$
\|v\|_{L^{2}(\mathcal{O} \times(0, T))} \leq \exp \left\{C(\Omega, \mathcal{O})\left(1+1 / T+\|g\|_{\infty}^{2 / 3}+T\|g\|_{\infty}\right)\right\}\left\|y^{0}\right\|_{L^{2}(\Omega)},
$$

where $g$ is given by (1.88).

This fixed point approach for the solution of controllability problems for nonlinear systems was introduced in [56] in the context of the controllability of the wave equation. Later, it was successfully applied to the semilinear heat equation in [36], [19], [30], [20], and [28].

The next case corresponds to a superlinear $f$, i.e., to a function not satisfying the last condition in (1.84). In this context, it is much more complicated to estimate the norms of $v_{z}$ and $y_{z}$, since $g$ is not necessarily uniformly bounded. However, something can still be made in some particular cases. More precisely, the following result is proved in [28].

TheOREM 1.7. Assume that

$$
\left\{\begin{array}{l}
f \in C^{1}(\mathbf{R}) \quad(\text { for instance }), \quad f(0)=0, \quad \text { and } \\
\frac{|f(s)|}{s \log ^{3 / 2}(1+|s|)} \longrightarrow 0 \quad \text { as }|s| \rightarrow+\infty .
\end{array}\right.
$$

Then (1.83) is null controllable with controls $v \in L^{\infty}(\mathcal{O} \times(0, T))$.

Remark 6. It is also proved in [28] that, in general, when

$$
|f(s)| \sim|s| \log ^{\beta}(1+|s|)
$$

for some $\beta>2,(1.83)$ is not null controllable. However, it is unknown whether or not the system (1.83) is null controllable when this is satisfied with $\frac{3}{2}<\beta \leq 2$. 
1.3.3. Another boundary conditions. Let us consider the following system, where the heat equation is completed with linear Robin (or Fourier) boundary conditions:

$$
\begin{cases}y_{t}-\Delta y=v 1_{\mathcal{O}} & \text { in } \quad Q, \\ \frac{\partial y}{\partial n}+a(x, t) y=0 & \text { on } \quad \Sigma, \\ y(0)=y^{0} & \text { in } \quad \Omega,\end{cases}
$$

where $a \in L^{\infty}(\Sigma)$ and $y^{0} \in L^{2}(\Omega)$. Recall that under these assumptions there exists a unique solution $y$ to (1.92), with

$$
y \in L^{2}\left(0, T ; H^{1}(\Omega)\right) \cap C^{0}\left([0, T] ; L^{2}(\Omega)\right) .
$$

Arguing as above, it is readily seen that the null controllability of (1.92) is implied by the observability of the adjoint system

$$
\begin{cases}-\varphi_{t}-\Delta \varphi=0 & \text { in } \quad Q \\ \frac{\partial \varphi}{\partial n}+a(x, t) \varphi=0 & \text { on } \quad \Sigma \\ \varphi(T)=\varphi^{0} & \text { in } \quad \Omega .\end{cases}
$$

An observability inequality for the solutions to (1.93) is proved in [30], under the additional assumption $a_{t} \in L^{\infty}(\Sigma)$. As a consequence, one also has the null controllability of (1.92) whenever $a$ fulfills this hypothesis. For some partial results concerning a nonlinear version of (1.92), see [15].

An improvement of these results has been obtained in the more recent work [21]. See section 2 for further details.

1.3.4. Another controllability problems. Another interesting problem is the null controllability of the heat equation with boundary controls, which means the null controllability of the system

$$
\begin{cases}y_{t}-\Delta y=0 & \text { in } \quad Q \\ y=v 1_{\gamma} & \text { on } \quad \Sigma \\ y(0)=y^{0} & \text { in } \quad \Omega\end{cases}
$$

when $\gamma$ is a (small) part of $\partial \Omega$. In this case, the adjoint system is again (1.2) but the required observability inequality is somewhat different. More precisely, we need the estimate

$$
\|\varphi(0)\|_{L^{2}(\Omega)}^{2} \leq C \iint_{\gamma \times(0, T)}\left|\frac{\partial \varphi}{\partial n}\right|^{2} d \sigma d t
$$

for all solutions to (1.2).

This can be obtained as a consequence of the following Carleman estimates, similar to (1.18) (see [30]):

$$
\begin{aligned}
& \iint_{Q} e^{-2 s \widetilde{\alpha}}\left((s \widetilde{\xi})^{-1}\left(\left|q_{t}\right|^{2}+|\Delta q|^{2}\right)+s \lambda^{2} \widetilde{\xi}|\nabla q|^{2}+s^{3} \lambda^{4} \widetilde{\xi}^{3}|q|^{2}\right) d x d t \\
& \quad \leq C\left(\iint_{Q} e^{-2 s \widetilde{\alpha}}\left|q_{t}+\Delta q\right|^{2} d x d t+s \iint_{\gamma \times(0, T)} e^{-2 s \widetilde{\alpha} \widetilde{\xi}}\left|\frac{\partial q}{\partial n}\right|^{2} d \sigma d t\right) .
\end{aligned}
$$


Here, $\widetilde{\alpha}=\widetilde{\alpha}(x, t)$ and $\widetilde{\xi}=\widetilde{\xi}(x, t)$ are appropriate weight functions, similar to $\alpha$ and $\xi$, respectively.

Using (1.95) and arguments like those in the proof of Theorem 1.1, one can easily deduce the null controllability of (1.94).

1.3.5. Other more general partial differential equations. The previous null controllability results can also be extended to more general equations with sufficiently regular coefficients. For instance, for the system

$$
\begin{cases}y_{t}-\partial_{i}\left(a_{i j}(x, t) \partial_{j} y\right)=v 1_{\mathcal{O}} & \text { in } Q, \\ y=0 & \text { on } \Sigma, \\ y(0)=y^{0} & \text { in } \Omega,\end{cases}
$$

where the coefficients $a_{i j} \in C^{2}(\bar{Q})$ are uniformly elliptic, it is proved in [30] that null controllability is achieved. This result has recently been improved in [51]; see subsection 2.2.3 below.

\section{Null controllability of other more general parabolic problems.}

2.1. The case of the linear heat equation with general zero and first order terms. In this subsection, we are going to prove the null controllability, with distributed controls, of the linear heat equation with zero and first order terms and coefficients in $L^{\infty}(Q)$, i.e.,

$$
\begin{cases}y_{t}-\Delta y+B(x, t) \cdot \nabla y+a(x, t) y=v 1_{\mathcal{O}} & \text { in } Q, \\ y=0 & \text { on } \Sigma, \\ y(0)=y^{0} & \text { in } \Omega,\end{cases}
$$

with $y^{0} \in L^{2}(\Omega), a \in L^{\infty}(Q)$, and $B \in L^{\infty}(Q)^{N}$. To achieve this, we will first prove an observability inequality for the associated adjoint system

$$
\begin{cases}-\varphi_{t}-\Delta \varphi-\nabla \cdot(\varphi B(x, t))+a(x, t) \varphi=0 & \text { in } Q, \\ \varphi=0 & \text { on } \Sigma, \\ \varphi(T)=\varphi^{0} & \text { in } \Omega .\end{cases}
$$

More precisely, we will prove that

$$
\|\varphi(0)\|_{L^{2}(\Omega)}^{2} \leq C \iint_{\mathcal{O} \times(0, T)}|\varphi|^{2} d x d t
$$

for all $\varphi^{0} \in L^{2}(\Omega)$ for some $C=C(\Omega, \mathcal{O}, T, a, B)$. To this end we will use the following lemma, which provides a suitable Carleman inequality (see [39]).

LEMmA 2.1. There exist $\lambda_{2}=C(\Omega, \mathcal{O}), s_{2}=C(\Omega, \mathcal{O})\left(T+T^{2}\right)$, and $C_{2}=$ $C_{2}(\Omega, \mathcal{O})>0$ such that, for any $\lambda \geq \lambda_{2}$ and any $s \geq s_{2}$, one has

$$
\begin{aligned}
& s \lambda^{2} \iint_{Q} e^{-2 s \alpha} \xi|\nabla q|^{2} d x d t+s^{3} \lambda^{4} \iint_{Q} e^{-2 s \alpha} \xi^{3}|q|^{2} d x d t \\
& \leq C_{2}\left(s^{3} \lambda^{4} \iint_{\mathcal{O} \times(0, T)} e^{-2 s \alpha} \xi^{3}|q|^{2} d x d t\right. \\
& \left.\quad+\iint_{Q} e^{-2 s \alpha}\left|F_{0}\right|^{2} d x d t+s^{2} \lambda^{2} \iint_{Q} e^{-2 s \alpha} \xi^{2}|F|^{2} d x d t\right)
\end{aligned}
$$


for all functions $q \in C^{1}(\bar{Q})$ with $q=0$ on $\Sigma$ and $q_{t}+\Delta q=F_{0}+\nabla \cdot F$, where $F_{0} \in$ $L^{2}(Q), F \in L^{2}(Q)^{N}$. Here, $\alpha$ and $\xi$ are the functions defined just before Lemma 1.3.

For the moment, let us assume that Lemma 2.1 is true and let us deduce an observability inequality of the kind (2.3) for the solutions to (2.2). Fixing $\lambda=\lambda_{2}$ and applying Lemma 2.1 to $\varphi$, we have

$$
\begin{aligned}
& s^{3} \iint_{Q} e^{-2 s \alpha} \xi^{3}|\varphi|^{2} d x d t+s \iint_{Q} e^{-2 s \alpha} \xi|\nabla \varphi|^{2} d x d t \\
& \leq C\left(s^{3} \iint_{\mathcal{O} \times(0, T)} e^{-2 s \alpha} \xi^{3}|\varphi|^{2} d x d t+\|a\|_{\infty}^{2} \iint_{Q} e^{-2 s \alpha}|\varphi|^{2} d x d t\right. \\
& \left.\quad+s^{2}\|B\|_{\infty}^{2} \iint_{Q} e^{-2 s \alpha} \xi^{2}|\varphi|^{2} d x d t\right)
\end{aligned}
$$

for any $s \geq s_{2}$. Consequently, we also have

$$
\iint_{Q} e^{-2 s \alpha} t^{-3}(T-t)^{-3}|\varphi|^{2} d x d t \leq C \iint_{\mathcal{O} \times(0, T)} e^{-2 s \alpha} t^{-3}(T-t)^{-3}|\varphi|^{2} d x d t
$$

for all $s \geq C\left(T+T^{2}+T^{2}\left(\|a\|_{\infty}^{2 / 3}+\|B\|_{\infty}^{2}\right)\right)$, whence it is easy to deduce that

$$
\iint_{\Omega \times(T / 4,3 T / 4)}|\varphi|^{2} d x d t \leq e^{C\left(1+1 / T+\|a\|_{\infty}^{2 / 3}+\|B\|_{\infty}^{2}\right)} \iint_{\mathcal{O} \times(0, T)}|\varphi|^{2} d x d t
$$

On the other hand, we also have (1.78) for the solutions to (2.2). This, together with (2.7), leads to the desired observability inequality (2.3). Thus, we have the following.

THEOREM 2.2. System (2.1) is null controllable with controls $v$ satisfying (1.80) and (1.81).

Following [39], let us now give the proof of the Carleman inequality (2.4).

Proof of Lemma 2.1. Let $q=q(x, t)$ satisfy the hypothesis stated above and set $q^{0}=\left.q\right|_{t=T}$. Then $q$ can be viewed as a solution by transposition of the backwards system

$$
\begin{cases}q_{t}+\Delta q=F_{0}+\nabla \cdot F & \text { in } Q, \\ q=0 & \text { on } \Sigma, \\ q(T)=q^{0} & \text { in } \Omega .\end{cases}
$$

In other words, for each $G \in L^{2}\left(0, T ; H^{-1}(\Omega)\right)$ we must have

$$
\int_{0}^{T}\langle G(t), q(t)\rangle d t=-\int_{0}^{T}\left\langle F_{0}(t)+\nabla \cdot F(t), z(t)\right\rangle d t+\left(q^{0}, z(T)\right)_{L^{2}(\Omega)},
$$

where $z$ is the solution of the linear problem

$$
\begin{cases}z_{t}-\Delta z=G & \text { in } \quad Q, \\ z=0 & \text { on } \quad \Sigma, \\ z(0)=0 & \text { in } \Omega .\end{cases}
$$

Here, $\langle\cdot, \cdot\rangle$ denotes the usual duality product between $H^{-1}(\Omega)$ and $H_{0}^{1}(\Omega)$. 
Let $s$ and $\lambda$ be as in Lemma 1.3 and let us introduce the following fourth order problem, which will be justified below:

$$
\begin{cases}\mathcal{L}\left(e^{-2 s \alpha} \mathcal{L}^{*} p\right)+s^{3} \lambda^{4} e^{-2 s \alpha} \xi^{3} q=-s^{3} \lambda^{4} e^{-2 s \alpha} \xi^{3} p 1_{\mathcal{O}} & \text { in } Q, \\ p=0, \quad e^{-2 s \alpha} \mathcal{L}^{*} p=0 & \text { on } \Sigma, \\ \left(e^{-2 s \alpha} \mathcal{L}^{*} p\right)(0)=\left(e^{-2 s \alpha} \mathcal{L}^{*} p\right)(T)=0 & \text { in } \Omega .\end{cases}
$$

Here, we have used the notation $\mathcal{L} q \equiv q_{t}-\Delta q, \quad \mathcal{L}^{*} q \equiv-q_{t}-\Delta q$. The partial differential problem (2.11) possesses exactly one (weak) solution $p$ with

$$
\iint_{Q} e^{-2 s \alpha}\left(\xi^{-1}\left(\left|p_{t}\right|^{2}+|\Delta p|^{2}\right)+\xi|\nabla p|^{2}+\xi^{3}|p|^{2}\right) d x d t<+\infty
$$

Indeed, let $P_{0}$ be the linear space

$$
P_{0}=\left\{z \in C^{2}(\bar{Q}): z=0 \text { on } \Sigma\right\}
$$

and let us set

$$
\left\{\begin{aligned}
& \kappa\left(p, p^{\prime}\right)=\iint_{Q} e^{-2 s \alpha} \mathcal{L}^{*} p \mathcal{L}^{*} p^{\prime} d x d t \\
&+s^{3} \lambda^{4} \iint_{\mathcal{O} \times(0, T)} e^{-2 s \alpha} \xi^{3} p p^{\prime} d x d t \quad \forall p, p^{\prime} \in P_{0}
\end{aligned}\right.
$$

and

$$
l(p)=-s^{3} \lambda^{4} \iint_{Q} e^{-2 s \alpha} \xi^{3} q p d x d t \quad \forall p \in P_{0} .
$$

Then $\kappa(\cdot, \cdot)$ is a positive and symmetric bilinear form in $P_{0}$.

Let $P$ be the completion of $P_{0}$ for the norm $\|p\|_{P}=(\kappa(p, p))^{1 / 2}$. Then $P$ is a Hilbert space for the scalar product $\kappa(\cdot, \cdot)$ and, in view of the Carleman inequality (1.18), we have that the functions in $P$ satisfy (2.12). It is also clear from (1.18) that $l$ is a continuous linear form on $P$ :

$$
|l(p)| \leq C\left(s^{3} \lambda^{4} \iint_{Q} e^{-2 s \alpha} \xi^{3}|q|^{2} d x d t\right)^{1 / 2}\|p\|_{P} \quad \forall p \in P .
$$

Consequently, from the Lax-Milgram lemma, the following variational equation possesses exactly one solution $p \in P$ :

$$
\kappa\left(p, p^{\prime}\right)=l\left(p^{\prime}\right) \quad \forall p^{\prime} \in P .
$$

It is not difficult to see that the unique solution to (2.17) also solves the fourth order problem (2.11) in the distributional sense.

Of course, the space $P$ and the function $p \in P$ depend on the choice we have made of $s$ and $\lambda$.

Now, let us set

$$
\widehat{z}=-e^{-2 s \alpha} \mathcal{L}^{*} p, \quad \widehat{u}=s^{3} \lambda^{4} e^{-2 s \alpha} \xi^{3} p 1_{\mathcal{O}} .
$$


It is readily seen from (2.11) that $\widehat{z}$ is, together with $\widehat{u}$, a solution to the null controllability problem

$$
\begin{cases}\widehat{z} t-\Delta \widehat{z}=s^{3} \lambda^{4} e^{-2 s \alpha} \xi^{3} q+\widehat{u} 1_{\mathcal{O}} & \text { in } Q, \\ \widehat{z}=0 & \text { on } \Sigma, \\ \widehat{z}(0)=\widehat{z}(T)=0 & \text { in } \Omega\end{cases}
$$

For the moment, we will assume that there exist positive $\widetilde{s}=\widetilde{s}(\Omega, \mathcal{O})$ and $\widetilde{\lambda}=\widetilde{\lambda}(\Omega, \mathcal{O})$, such that

$$
\begin{aligned}
& s^{-3} \lambda^{-4} \iint_{\mathcal{O} \times(0, T)} e^{2 s \alpha} \xi^{-3}|\hat{u}|^{2} d x d t+\iint_{Q} e^{2 s \alpha}|\hat{z}|^{2} d x d t \\
& \quad+s^{-2} \lambda^{-2} \iint_{Q} e^{2 s \alpha} \xi^{-2}|\nabla \hat{z}|^{2} d x d t \leq C s^{3} \lambda^{4} \iint_{Q} e^{-2 s \alpha} \xi^{3}|q|^{2} d x d t
\end{aligned}
$$

for all $s \geq \widetilde{s}\left(T+T^{2}\right)$ and $\lambda \geq \widetilde{\lambda}$.

Let us then prove (2.4). First, we will get an estimate for the second term on the left-hand side of (2.4). In view of (2.9) written for $G=s^{3} \lambda^{4} e^{-2 s \alpha} \xi^{3} q+\widehat{u} 1_{\mathcal{O}}$, we have

$$
s^{3} \lambda^{4} \iint_{Q} e^{-2 s \alpha} \xi^{3}|q|^{2} d x d t=-\iint_{\mathcal{O} \times(0, T)} q \widehat{u} d x d t+\int_{0}^{T}\left\langle F_{0}(t)+\nabla \cdot F(t), \widehat{z}(t)\right\rangle d t .
$$

Therefore,

$$
\begin{aligned}
s^{3} \lambda^{4} & \iint_{Q} e^{-2 s \alpha} \xi^{3}|q|^{2} d x d t \\
\leq & \left(\int_{0}^{T} \int_{\mathcal{O}} e^{-2 s \alpha} \xi^{3}|q|^{2} d x d t\right)^{1 / 2}\left(\int_{0}^{T} \int_{\mathcal{O}} e^{2 s \alpha} \xi^{-3}|\widehat{u}|^{2} d x d t\right)^{1 / 2} \\
& +\left(\iint_{Q} e^{-2 s \alpha}\left|F_{0}\right|^{2} d x d t\right)^{1 / 2}\left(\iint_{Q} e^{2 s \alpha}|\widehat{z}|^{2} d x d t\right)^{1 / 2} \\
& +\left(\iint_{Q} e^{-2 s \alpha} \xi^{2}|F|^{2} d x d t\right)^{1 / 2}\left(\iint_{Q} e^{2 s \alpha} \xi^{-2}|\nabla \widehat{z}|^{2} d x d t\right)^{1 / 2}
\end{aligned}
$$

And now, using (2.20), we see that

$$
\begin{aligned}
& s^{3} \lambda^{4} \iint_{Q} e^{-2 s \alpha} \xi^{3}|q|^{2} d x d t \leq C\left(s^{3} \lambda^{4} \iint_{\mathcal{O} \times(0, T)} e^{-2 s \alpha} \xi^{3}|q|^{2} d x d t\right. \\
& \left.\quad+\iint_{Q} e^{-2 s \alpha}\left|F_{0}\right|^{2} d x d t+s^{2} \lambda^{2} \iint_{Q} e^{-2 s \alpha} \xi^{2}|F|^{2} d x d t\right)
\end{aligned}
$$

for $s \geq \widehat{s}\left(T+T^{2}\right)$ and $\lambda \geq \widehat{\lambda}$.

Let us now get an estimate for the first term on the left-hand side of (2.4). To this end, we multiply by $s \lambda^{2} e^{-2 s \alpha} \xi q$ the equation satisfied by $q$ and integrate in space 
and time. This gives

$$
\begin{aligned}
s \lambda^{2} \iint_{Q} e^{-2 s \alpha} \xi|\nabla q|^{2} d x d t+ & s \frac{\lambda^{2}}{2} \iint_{Q}\left(\left(e^{-2 s \alpha} \xi\right)_{t}-\Delta\left(e^{-2 s \alpha} \xi\right)\right)|q|^{2} d x d t \\
= & s \lambda^{2} \iint_{Q} e^{-2 s \alpha}\left(\xi F_{0} q-\xi F \cdot \nabla q\right) d x d t \\
& -s \lambda^{2} \iint_{Q} F \cdot \nabla\left(e^{-2 s \alpha} \xi\right) q d x d t
\end{aligned}
$$

Then we use the estimates

$$
\begin{aligned}
\left|\left(e^{-2 s \alpha} \xi\right)_{t}\right| & \leq C e^{-2 s \alpha}\left(s T \xi^{3}+T \xi^{2}\right) \leq C s^{2} e^{-2 s \alpha} \xi^{3}, \\
\left|\Delta\left(e^{-2 s \alpha} \xi\right)\right| & \leq C s^{2} \lambda^{2} e^{-2 s \alpha} \xi^{3}
\end{aligned}
$$

for $s \geq C\left(T+T^{2}\right)$,

$$
\begin{aligned}
s \lambda^{2}\left|\iint_{Q} e^{-2 s \alpha} \xi F_{0} q d x d t\right| \leq & C\left(s^{3} \lambda^{4} \iint_{Q} e^{-2 s \alpha} \xi^{3}|q|^{2} d x d t\right. \\
& \left.+s^{-1} \iint_{Q} e^{-2 s \alpha} \xi^{-1}\left|F_{0}\right|^{2} d x d t\right), \\
s \lambda^{2}\left|\iint_{Q} e^{-2 s \alpha} \xi F \cdot \nabla q d x d t\right| \leq & C s \lambda^{2} \iint_{Q} e^{-2 s \alpha} \xi|F|^{2} d x d t \\
& +\frac{1}{2} s \lambda^{2} \iint_{Q} e^{-2 s \alpha} \xi|\nabla q|^{2} d x d t,
\end{aligned}
$$

and

$$
\begin{aligned}
& s \lambda^{2}\left|\iint_{Q} F \cdot \nabla\left(e^{-2 s \alpha} \xi\right) q d x d t\right| \\
& \quad \leq C\left(s \lambda^{2} \iint_{Q} e^{-2 s \alpha} \xi|F|^{2} d x d t+s^{3} \lambda^{4} \iint_{Q} e^{-2 s \alpha} \xi^{3}|q|^{2} d x d t\right),
\end{aligned}
$$

to obtain from (2.24) the following:

$$
\begin{aligned}
& s \lambda^{2} \iint_{Q} e^{-2 s \alpha} \xi|\nabla q|^{2} d x d t \leq C\left(s^{3} \lambda^{4} \iint_{Q} e^{-2 s \alpha} \xi^{3}|q|^{2} d x d t\right. \\
& \left.\quad+\iint_{Q} e^{-2 s \alpha}\left|F_{0}\right|^{2} d x d t+s \lambda^{2} \iint_{Q} e^{-2 s \alpha} \xi|F|^{2} d x d t\right)
\end{aligned}
$$

for $s \geq C\left(T+T^{2}\right)$. This, together with (2.23), provides (2.4).

Let us finally prove $(2.20)$.

We first multiply the equation in (2.11) by $p$, so we have

$$
\chi(p, p)=-s^{3} \lambda^{4} \iint_{Q} e^{-2 s \alpha} \xi^{3} q p d x d t,
$$

which, combined with the Carleman inequality (1.18) proved in Lemma 1.3, provides the desired inequality for the two first terms on the left-hand side of $(2.20)$. 
To estimate the first order term, we multiply by $s^{-2} \lambda^{-2} e^{2 s \alpha} \xi^{-2} \widehat{z}$ the equation verified by $\widehat{z}$. Then we integrate by parts with respect to the space variable and get

$$
\begin{gathered}
s^{-2} \lambda^{-2} \iint_{Q} e^{2 s \alpha} \xi^{-2} \widehat{z} \widehat{z}_{t} d x d t+s^{-2} \lambda^{-2} \iint_{Q} e^{2 s \alpha} \xi^{-2}|\nabla \widehat{z}|^{2} d x d t \\
-2 s^{-1} \lambda^{-1} \iint_{Q} e^{2 s \alpha} \xi^{-1} \nabla \eta^{0} \cdot \nabla \widehat{z} \widehat{z} d x d t \\
\quad-2 s^{-2} \lambda^{-1} \iint_{Q} e^{2 s \alpha} \xi^{-2} \nabla \eta^{0} \cdot \nabla \widehat{z} \widehat{z} d x d t \\
=s \lambda^{2} \iint_{Q} \xi q \widehat{z}+s^{-2} \lambda^{-2} \iint_{\mathcal{O} \times(0, T)} e^{2 s \alpha} \xi^{-2} \widehat{u} \widehat{z} d x d t
\end{gathered}
$$

This time, let us integrate by parts in the first term with respect to the time variable:

$$
\begin{aligned}
& s^{-2} \lambda^{-2} \iint_{Q} e^{2 s \alpha} \xi^{-2} \widehat{z} \widehat{z}_{t} d x d t \\
& \quad=-\frac{1}{2} s^{-2} \lambda^{-2} \iint_{Q}\left(e^{2 s \alpha} \xi^{-2}\right)_{t}|\widehat{z}|^{2} d x d t \\
& \quad \leq C s^{-1} \lambda^{-2} T \iint_{Q} e^{2 s \alpha}|\widehat{z}|^{2} d x d t \leq C \iint_{Q} e^{2 s \alpha}|\widehat{z}|^{2} d x d t
\end{aligned}
$$

for $s \geq C T$ and $\lambda \geq 1$.

Finally, we use Young's inequality for the other terms of (2.30) and obtain

$$
\begin{gathered}
-2 s^{-1} \lambda^{-1} \iint_{Q} e^{2 s \alpha} \xi^{-1} \nabla \eta^{0} \cdot \nabla \widehat{z} \widehat{z} d x d t \\
-2 s^{-2} \lambda^{-1} \iint_{Q} e^{2 s \alpha} \xi^{-2} \nabla \eta^{0} \cdot \nabla \widehat{z} \widehat{z} d x d t \\
\leq C \iint_{Q} e^{2 s \alpha}|\widehat{z}|^{2} d x d t+\frac{1}{2} s^{-2} \lambda^{-2} \iint_{Q} e^{2 s \alpha} \xi^{-2}|\nabla \widehat{z}|^{2} d x d t \\
s \lambda^{2} \iint_{Q} \xi q \widehat{z} \leq C\left(\iint_{Q} e^{2 s \alpha}|\widehat{z}|^{2} d x d t+s^{3} \lambda^{4} \iint_{Q} e^{-2 s \alpha} \xi^{3}|q|^{2} d x d t\right),
\end{gathered}
$$

and

$$
\begin{aligned}
& s^{-2} \lambda^{-2} \iint_{\mathcal{O} \times(0, T)} e^{2 s \alpha} \xi^{-2} \widehat{u} \widehat{z} d x d t \\
& \quad \leq C\left(\iint_{Q} e^{2 s \alpha}|\widehat{z}|^{2} d x d t+s^{-3} \lambda^{-4} \iint_{\mathcal{O} \times(0, T)} e^{2 s \alpha} \xi^{-3}|\widehat{u}|^{2} d x d t\right)
\end{aligned}
$$

for $s \geq C T^{2}$.

As a conclusion, we deduce (2.20) directly from (2.30).

Remark 7 . In the same spirit of Remark 4, it would be very interesting to know whether or not the powers of $s$ and $\lambda$ in (2.4) are optimal. 
2.2. Null controllability of other semilinear problems and further comments. With the help of Lemma 2.1, Theorem 2.2, and some variants, we can now prove the null controllability of other nonlinear parabolic systems. This is the goal of this section.

2.2.1. Null controllability of the semilinear heat equation with nonlinearities in the zero and first order terms. In this paragraph, we will deduce the null controllability of the system

$$
\begin{cases}y_{t}-\Delta y+f(y, \nabla y)=v 1_{\mathcal{O}} & \text { in } Q \\ y=0 & \text { on } \Sigma, \\ y(0)=y^{0} & \text { in } \Omega,\end{cases}
$$

where we suppose $y^{0} \in H_{0}^{1}(\Omega) \cap W^{1, \infty}(\Omega), f \in C^{1}\left(\mathbf{R} \times \mathbf{R}^{N}\right), f(0,0)=0$, and

$$
f(s, p)=g(s, p) s+G(s, p) \cdot p \quad \forall(s, p) \in \mathbf{R} \times \mathbf{R}^{N}
$$

for some functions $g$ and $G$ satisfying $|g| \leq C$ and $|G| \leq C$.

To this end, we will combine Theorem 2.2 and a fixed point argument.

Thus, let us consider the map

$$
z \in L^{2}\left(0, T ; H_{0}^{1}(\Omega)\right) \mapsto y_{z} \in L^{2}\left(0, T ; H_{0}^{1}(\Omega)\right),
$$

where $\left(y_{z}, v_{z}\right)$ is the solution of

$$
\begin{cases}\left(y_{z}\right)_{t}-\Delta y_{z}+g(z, \nabla z) y_{z}+G(z, \nabla z) \cdot \nabla y_{z}=v_{z} 1_{\mathcal{O}} & \text { in } Q, \\ y_{z}=0 & \text { on } \Sigma, \\ y_{z}(0)=y^{0}, \quad y_{z}(T)=0 & \text { in } \Omega,\end{cases}
$$

constructed as in the previous subsection. We know that

$$
\left\|v_{z}\right\|_{L^{2}(\mathcal{O} \times(0, T))} \leq K\left\|y^{0}\right\|_{L^{2}(\Omega)},
$$

with a constant $K$ of the form

$$
K=\exp \left\{C\left(1+\frac{1}{T}+\|g\|_{\infty}^{2 / 3}+T\|g\|_{\infty}+(1+T)\|G\|_{\infty}^{2}\right)\right\} .
$$

Now, one can check from parabolic regularity and the fact that $g$ and $G$ are uniformly bounded that Kakutani's fixed point theorem is applicable. For convenience, let us state this result.

TheOREM 2.3. Let $Z$ be a Banach space and let $\Lambda: Z \mapsto Z$ be a set-valued mapping satisfying the following assumptions:

1. $\Lambda(z)$ is a nonempty closed convex set of $Z$ for every $z \in Z$.

2. There exists a nonempty convex compact set $K \subset Z$ such that $\Lambda(K) \subset K$.

3. $\Lambda$ is upper-hemicontinuous in $Z$, i.e., for each $\sigma \in Z^{\prime}$ the single-valued mapping

$$
z \mapsto \sup _{y \in \Lambda(z)}\langle\sigma, y\rangle_{Z^{\prime}, Z}
$$

is upper-semicontinuous. 
Then $\Lambda$ possesses a fixed point in the set $K$, i.e., there exists $z \in K$ such that $z \in$ $\Lambda(z)$.

For the proof, see, for instance, [3]. We then have the following result.

THEOREM 2.4. Under the previous assumptions on $y^{0}$ and $f,(2.35)$ is null controllable with controls $v$ satisfying

$$
\|v\|_{L^{2}(\mathcal{O} \times(0, T))} \leq K\left\|y^{0}\right\|_{L^{2}(\Omega)}
$$

where the constant $K$ is given by (2.40).

Now, we pretend to extend this result to the (superlinear) case where we only have $f \in C^{1}\left(\mathbf{R} \times \mathbf{R}^{N}\right)$ and $f(0,0)=0$. This time, to apply similar arguments, one must work in the space $L^{\infty}\left(0, T ; W^{1, \infty}(\Omega)\right)$ instead of $L^{2}\left(0, T ; H_{0}^{1}(\Omega)\right)$. In fact, we have the following result (see [16]).

Theorem 2.5. Assume that $f \in C^{1}\left(\mathbf{R} \times \mathbf{R}^{N}\right), f(0,0)=0$, and (2.36) holds with

$$
\frac{g(s, p)}{\log ^{3 / 2}(1+|s|+|p|)} \rightarrow 0 \quad \text { and } \quad \frac{G(s, p)}{\log ^{1 / 2}(1+|s|+|p|)} \rightarrow 0
$$

as $|s| \rightarrow+\infty$. Then (2.35) is null controllable with controls in $L^{\infty}(\mathcal{O} \times(0, T))$.

Remark 8. As in Theorem 1.7, it is unknown whether system (2.35) is controllable when

$$
|g(s, p)| \sim \log ^{\beta}(1+|s|+|p|) \quad \text { and/or } \quad|G(s, p)| \sim \log ^{\gamma}(1+|s|+|p|)
$$

with $\beta>\frac{3}{2}, \gamma>\frac{1}{2}$.

Let us now address other typical controllability problems in this context.

(A) Going back to system (1.83), with no hypothesis on the growth of $f$, the null controllability can be achieved if $f$ possesses the "good sign," that is to say, if $f(s) s \geq 0$ for all $s \in \mathbf{R}$.

In this situation, it can be proved that, for any $\rho>0$, there exists a time $T=$ $T(\Omega, \mathcal{O}, f, \rho)>0$ such that, for every $y^{0} \in L^{2}(\Omega)$ with $\left\|y^{0}\right\|_{L^{2}(\Omega)} \leq \rho$, there exists a control $v \in L^{2}(\mathcal{O} \times(0, T))$ whose associated solution $y$ verifies $y(T)=0$ (see [2], [5], and [28] for more details).

(B) Using methods similar to those above, the exact controllability to the trajectories can be deduced as well. For instance, the following result is proved in [16].

Theorem 2.6. Let us assume that $y^{0} \in H_{0}^{1}(\Omega) \cap W^{1, \infty}(\Omega), f \in C^{1}\left(\mathbf{R} \times \mathbf{R}^{N}\right)$,

$$
\frac{1}{\log ^{3 / 2}(1+|s|+|p|)}\left|\int_{0}^{1} \frac{\partial f}{\partial s}\left(s_{0}+\lambda s, p_{0}+\lambda p\right) d \lambda\right| \rightarrow 0
$$

and

$$
\frac{1}{\log ^{1 / 2}(1+|s|+|p|)}\left|\int_{0}^{1} \frac{\partial f}{\partial p_{i}}\left(s_{0}+\lambda s, p_{0}+\lambda p\right) d \lambda\right| \rightarrow 0
$$

for $1 \leq i \leq N$ as $|(s, p)| \rightarrow+\infty$, uniformly in $\left(s_{0}, p_{0}\right) \in K$ for every compact set $K \subset \mathbf{R} \times \mathbf{R}^{N}$. Let $y_{*}^{0} \in H_{0}^{1}(\Omega) \cap W^{1, \infty}(\Omega)$ and $v_{*} \in L^{\infty}(\mathcal{O} \times(0, T))$ be given and let $y_{*} \in C\left([0, T] ; W^{1, \infty}(\Omega)\right)$ be the corresponding solution to (2.35). Then there exists $v \in L^{\infty}(\mathcal{O} \times(0, T))$ such that its associated solution y verifies $y(T)=y_{*}(T)$. 
2.2.2. Heat equations with Fourier boundary conditions. In this paragraph, we will recall some recent results concerning the null and exact controllability to the trajectories of the following partial differential systems:

$$
\begin{cases}y_{t}-\Delta y+B \cdot \nabla y+a y=v 1_{\mathcal{O}} & \text { in } \quad Q \\ \frac{\partial y}{\partial n}+\beta y=0 & \text { on } \Sigma \\ y(0)=y^{0} & \text { in } \Omega\end{cases}
$$

and

$$
\begin{cases}y_{t}-\Delta y+F(y, \nabla y)=v 1_{\mathcal{O}} & \text { in } \quad Q, \\ \frac{\partial y}{\partial n}+f(y)=0 & \text { on } \Sigma, \\ y(0)=y^{0} & \text { in } \Omega,\end{cases}
$$

where $y^{0} \in L^{2}(\Omega)$,

$$
a \in L^{\infty}(Q), \quad B \in L^{\infty}(Q)^{N}, \quad \beta \in L^{\infty}(\Sigma),
$$

and (for instance)

$$
F \in C^{1}\left(\mathbf{R} \times \mathbf{R}^{N}\right), \quad f \in C^{1}(\mathbf{R}) .
$$

Let us first deduce a null controllability result for (2.47). To this end, we will need, as before, an observability estimate for the solutions to the adjoint system associated to $(2.47)$.

Thus, let us consider the backwards system

$$
\begin{cases}-\varphi_{t}-\Delta \varphi-\nabla \cdot(\varphi B(x, t))+a(x, t) \varphi=0 & \text { in } Q \\ (\nabla \varphi+\varphi B(x, t)) \cdot n+\beta(x, t) \varphi=0 & \text { on } \Sigma \\ \varphi(T)=\varphi^{0} & \text { in } \Omega\end{cases}
$$

where $\varphi^{0} \in L^{2}(\Omega)$. For the solutions to these problems, one has the following Carleman inequality (see [21]):

$$
\iint_{Q} e^{-2 s \zeta} \kappa^{3}|\varphi|^{2} d x d t \leq C \iint_{\mathcal{O} \times(0, T)} e^{-2 s \zeta} \kappa^{3}|\varphi|^{2} d x d t
$$

for appropriate positive weight functions $\kappa$ and $\zeta$ (similar to those defined in section 1) depending on a parameter $\lambda$ and a suitable choice of $s$ and $\lambda$. The proof of (2.52) can be achieved using duality arguments similar to those presented in the proof of Lemma 2.1.

From (2.52), one can easily deduce an observability inequality for the solutions to (2.51). This yields the next null controllability result.

TheOREm 2.7. Let (2.49) be fulfilled. Then, for all $y^{0} \in L^{2}(\Omega)$, there exists a control $v \in L^{\infty}(\mathcal{O} \times(0, T))$ such that the associated solution to (2.47) verifies

$$
y(T)=0 \quad \text { in } \Omega .
$$


Finally, the same arguments used in [28], based on the explicit dependence of the constant $C$ in (2.52) with respect to $T$ and the $L^{\infty}$-norms of the coefficients $a, B$, and $\beta$, lead to an exact controllability result to the trajectories for the nonlinear system (2.48).

THEOREM 2.8. Let (2.50) be fulfilled and let $\bar{y}$ be a solution of the nonlinear system (2.48) with $v \equiv 0$ such that

$$
\bar{y} \in L^{2}\left(0, T ; H^{1}(\Omega)\right) \cap L^{\infty}(Q), \quad \bar{y}(0) \in L^{\infty}(\Omega) .
$$

Moreover, assume that the functions $F$ and $f$ verify

$$
\lim _{|s| \rightarrow \infty} \frac{|F(s, p)-F(r, p)|}{|s-r| \log ^{3 / 2}(1+|s-r|)}=0
$$

uniformly in $(r, p) \in \mathbf{R} \times \mathbf{R}^{N}$,

$$
\left\{\begin{array}{l}
\forall L>0, \exists M>0 \text { such that } \\
|F(s, p)-F(r, p)| \leq M|s-r|, \quad|F(s, p)-F(s, q)| \leq M|p-q| \\
\forall(s, r, p, q) \in[-L, L]^{2} \times \mathbf{R}^{N} \times \mathbf{R}^{N}
\end{array}\right.
$$

and

$$
\lim _{|s| \rightarrow \infty} \frac{|f(s)-f(r)|}{|s-r| \log ^{1 / 2}(1+|s-r|)}=0
$$

uniformly in $r \in \mathbf{R}$. Then there exist controls $v \in L^{\infty}(\mathcal{O} \times(0, T))$ and associated solutions $y$ to (2.48) such that $y(T)=\bar{y}(T)$.

For the proof of this result and other related questions, see [22].

2.2.3. Null controllability of some variants of the heat system. Here, we are interested in solving the null controllability problem of more general parabolic systems, where various coefficients appear in the differential equation.

We first consider the case of a general second order differential operator in the heat system - more precisely, the following problem:

$$
\begin{cases}y_{t}-\sum_{i, j=1}^{N} \partial_{i}\left(a_{i j} \partial_{j} y\right)=v 1_{\mathcal{O}} & \text { in } \quad Q \\ y=0 & \text { on } \Sigma, \\ y(0)=y^{0} & \text { in } \Omega,\end{cases}
$$

where $y^{0} \in L^{2}(\Omega)$ and the coefficients $a_{i j}=a_{j i} \in W^{1, \infty}(Q)(1 \leq i, j \leq N)$ form a uniformly elliptic matrix. For the solutions to the adjoint system associated to (2.57) (which is in fact the same), we find a global Carleman inequality in the recent paper [51]. Of course, this leads to the null controllability of (2.57).

Analogously as we found in Theorem 2.2, this allows us to deduce a null controllability result for (2.57) in a more general case.

THEOREM 2.9. Let us assume that, in (2.57), the coefficients $a_{i j}$ are of the form $a_{i j}=a_{i j}^{0}+\varepsilon h_{i j}$ with $a_{i j}^{0}=a_{j i}^{0} \in W^{1, \infty}(Q)$ uniformly elliptic, $(T-t)^{-1 / 2} h_{i j} \in L^{\infty}(Q)$ and $\varepsilon$ small enough, depending on $\Omega, \mathcal{O}, T, a_{i j}^{0}$, and $h_{i j}$. Then, for any $T>0$, system (2.57) is null controllable at time $T$. 
For the proof of Theorem 2.9, it suffices to combine the previously mentioned result for general $a_{i j} \in W^{1, \infty}(Q)$ with Lemma 2.1 adapted to this situation.

The second case we consider is the following:

$$
\begin{cases}y_{t}-\sum_{i, j=1}^{N} a_{i j} \partial_{i j} y=v 1_{\mathcal{O}} & \text { in } Q, \\ y=0 & \text { on } \Sigma, \\ y(0)=y^{0} & \text { in } \Omega,\end{cases}
$$

with $y^{0} \in L^{2}(\Omega)$. To prove the null controllability of (2.58), we can use a recent Carleman inequality which can be found in [23] for heat equations with right-hand sides in $L^{2}\left(0, T ; H^{-2}(\Omega)\right)$. This is given in the following result.

LEMMA 2.10. Let us consider the following problem:

$$
\begin{cases}-z_{t}-\Delta z=\sum_{i, j=1}^{N} \partial_{i j} H_{i j} & \text { in } Q, \\ z=0 & \text { on } \Sigma, \\ z(T)=z^{0} & \text { in } \Omega,\end{cases}
$$

where $z_{0} \in H^{-1}(\Omega)$ and the functions $H_{i j}$ verify $H_{i j} \in L^{2}(Q)$ with

$$
\sum_{j=1}^{N} \partial_{j} H_{i j} \in L^{2}(Q) \quad \text { and } \quad \sum_{j=1}^{N} H_{i j} n_{j}=0 .
$$

There exist constants $C_{3}(\Omega, \mathcal{O})>0, \lambda_{3}=C(\Omega, \mathcal{O})$, and $s_{3}=C(\Omega, \mathcal{O})\left(T+T^{2}\right)$ such that

$$
\begin{aligned}
s^{3} \lambda^{4} \iint_{Q} e^{-2 s \alpha} \xi^{3}|z|^{2} d x d t \leq & C_{3}\left(s^{3} \lambda^{4} \iint_{\mathcal{O} \times(0, T)} e^{-2 s \alpha} \xi^{3}|z|^{2} d x d t\right. \\
& \left.+s^{4} \lambda^{4} \sum_{i, j=1}^{N} \iint_{Q} e^{-2 s \alpha} \xi^{4}\left|H_{i j}\right|^{2} d x d t\right)
\end{aligned}
$$

for all $\lambda \geq \lambda_{3}$ and $s \geq s_{3}$.

Let us introduce the adjoint system to (2.58):

$$
\begin{cases}-\varphi_{t}-\partial_{i j}\left(a_{i j} \varphi\right)=0 & \text { in } Q, \\ \varphi=0 & \text { on } \Sigma, \\ \varphi(T)=\varphi^{0} & \text { in } \Omega .\end{cases}
$$

A simple application of Lemma 2.10 gives the following result.

THEOREM 2.11. Let $T$ be an arbitrary positive time. Let us suppose that $a_{i j}=$ $\delta_{i j}+\varepsilon h_{i j}(i, j=1, \ldots, N)$ with $(T-t)^{-1 / 2} h_{i j} \in L^{\infty}(Q)$ and $\varepsilon>0$ small enough, depending on $\Omega, \mathcal{O}$, and $T$. Then (2.58) is null controllable at time $T$.

Let us finally consider the control problem

$$
\begin{cases}b y_{t}-\Delta y=v 1_{\mathcal{O}} & \text { in } \quad Q, \\ y=0 & \text { on } \Sigma, \\ y(0)=y^{0} & \text { in } \Omega,\end{cases}
$$


whose adjoint system is the following:

$$
\begin{cases}-(b \varphi)_{t}-\Delta \varphi=0 & \text { in } Q \\ \varphi=0 & \text { on } \Sigma \\ \varphi(T)=\varphi^{0} & \text { in } \Omega .\end{cases}
$$

In order to analyze the null controllability of (2.63), we need a Carleman inequality for heat equations with right-hand sides in $H^{-1}\left(0, T ; L^{2}(\Omega)\right)$. Its proof can also be found in [23].

Lemma 2.12. Consider the system

$$
\left\{\begin{array}{l}
-z_{t}-\Delta z=G_{t} Q \\
z=0 \Sigma \\
z(T)=z^{0} \Omega
\end{array}\right.
$$

where $z^{0} \in H^{-1}(\Omega)$ and $G \in L^{2}(Q)$ with $G \in C_{w}\left([0, T] ; H^{-1}(\Omega)\right)$. Then there exist $C_{4}(\Omega, \mathcal{O})>0, \lambda_{4}=C(\Omega, \mathcal{O})$, and $s_{4}=C(\Omega, \mathcal{O})\left(T+T^{2}\right)$ such that

$$
\begin{array}{r}
s^{3} \lambda^{4} \iint_{Q} e^{-2 s \alpha} \xi^{3}|z|^{2} d x d t \leq C_{4}\left(s^{3} \lambda^{4} \iint_{\mathcal{O} \times(0, T)} e^{-2 s \alpha} \xi^{3}|z|^{2} d x d t\right. \\
\left.+s^{4} \lambda^{4} \iint_{Q} e^{-2 s \alpha} \xi^{4}|G|^{2} d x d t\right)
\end{array}
$$

for all $\lambda \geq \lambda_{4}$ and $s \geq s_{4}$.

From this Carleman inequality, one can readily deduce the following result.

THEOREM 2.13. Let $T$ be a positive time and let us assume that $b=1+\varepsilon h$ with $(T-t)^{-1 / 2} h \in L^{\infty}(Q)$ and $\varepsilon>0$ small enough, depending on $\Omega, \mathcal{O}$, and $T$. Then system (2.63) is null controllable at time $T$.

Remark 9. The proofs of Lemmas 2.10 and 2.12 are based on the duality arguments presented in Lemma 2.1. Observe in particular that the solutions to systems (2.58) and (2.63) belong only to $L^{2}(Q)$ and must be defined by transposition.

3. Null controllability of systems of the Stokes kind. In this section, $N=$ 2 or $N=3$. The controllability properties of the Navier-Stokes system have been the subject of intensive research these last years. The question was first considered by Lions in [48], where approximate controllability was conjectured. This was followed by several papers, where various partial (positive) answers were furnished. See [18], [10], [11], and [50]. Concerning null controllability and exact controllability to the trajectories, the first local results were given in [29].

For completeness, we will recall briefly one of the main results in [10]. This paper deals with the approximate controllability of the two-dimensional Navier-Stokes equations with Navier-slip boundary conditions on the lateral boundary $\partial \Omega \times(0, T)$. Thus, let us assume that the state equation is

$$
\begin{cases}y_{t}+(y \cdot \nabla) y-\Delta y+\nabla p=v 1_{\mathcal{O}}, \quad \nabla \cdot y=0 & \text { in } Q, \\ y \cdot n=0, \quad \nabla \times y=0 & \text { on } \Sigma, \\ y(0)=y^{0} & \text { in } \Omega\end{cases}
$$

(where $\Omega \subset \mathbf{R}^{2}$ ). The general fact the author makes good use of is the treatment of $(y \cdot \nabla) y$ as the leading term, while $-\Delta y$ is considered as a "disturbance." 
Roughly speaking, the following is proved: Let $y^{0}$ and $y^{1}$ be two prescribed velocity fields. Then the fluid can be driven from $y^{0}$ at time $t=0$ to a velocity field at time $t=T$ which is arbitrarily close to $y^{1}$. Here, "arbitrarily close" means that, for instance, the distance of the velocity field to $y^{1}$ in $W^{-1, \infty}(\Omega)$ and the distance of the vorticity to $\nabla \times y_{1}$ in all spaces $L^{\infty}(K)(K \subset \Omega$ is an arbitrary compact set) can be made arbitrarily small. This is proved for any $T>0$, for any regular $\Omega, y^{0}$, and $y^{1}$, and for all nonempty open sets $\mathcal{O} \subset \Omega$.

The proof is as follows:

1. For each $\alpha>0$, it is possible to find $v_{\alpha}$ and a couple $\left(y_{\alpha}, p_{\alpha}\right)$ such that $y_{\alpha}$ is a gradient outside $\mathcal{O} \times(0, T)$,

$$
\begin{cases}y_{\alpha, t}+\left(y_{\alpha} \cdot \nabla\right) y_{\alpha}-\Delta y_{\alpha}+\nabla p_{\alpha}=v_{\alpha} 1_{\mathcal{O}}, \nabla \cdot y_{\alpha}=0 & \text { in } Q, \\ y_{\alpha} \cdot n=0, \quad \nabla \times y_{\alpha}=0 & \text { on } \Sigma, \\ y_{\alpha}(0)=y_{\alpha}(T)=0 & \text { in } \Omega\end{cases}
$$

and, furthermore, the linearized Euler system at $y_{\alpha}$, that is to say,

$$
\begin{cases}z_{t}+\left(y_{\alpha} \cdot \nabla\right) z+(z \cdot \nabla) y_{\alpha}+\nabla \pi=w 1_{\mathcal{O}}, \quad \nabla \cdot z=0 & \text { in } Q \\ z \cdot n=0, \quad \nabla \times z=0 & \text { on } \Sigma,\end{cases}
$$

is $\alpha$-controllable in the following sense: For any given $z_{0}$ and $z_{d}$ of class $C^{\infty}$, there exists a control $w$ such that $(3.3)$ possesses at least one solution bounded in $C^{3}(\bar{Q})$ independently of $\alpha$ and satisfying

$$
z(0)=z_{0} \text { in } \Omega
$$

and

$$
z(T)=z_{d} \text { in }\{x \in \Omega ; \operatorname{dist}(x, \partial \Omega) \geq \alpha\}
$$

(notice we are saying that, for every $\alpha$, this property is satisfied for any $z_{0}$ and $z_{d}$ ).

2. Let us set $v(x, t)=0$ in (3.1) for $t \in[0,(1-\delta) T]$ ( $\delta$ will be determined below). This defines $y$ and $p$ without ambiguity in $\Omega \times[0,(1-\delta) T]$ and, in particular, we can speak of $y((1-\delta) T)$. In $[(1-\delta) T, T]$, we do the following:

- First, $v_{\alpha}, y_{\alpha}$, and $p_{\alpha}$ are rescaled. In view of the $\alpha$-controllability of (3.3), we introduce a first control function $\widetilde{v}$ :

$$
\widetilde{v}(x, t) \equiv \frac{1}{\delta} v_{\alpha}\left(x, \frac{1}{\delta}(t-(1-\delta) T)\right)+w\left(x, \frac{1}{\delta}(t-(1-\delta) T)\right) .
$$

The associate state is

$$
(\widetilde{y}, \widetilde{p}) \equiv \frac{1}{\delta}\left(y_{\alpha}, p_{\alpha}\right)\left(x, \frac{1}{\delta}(t-(1-\delta) T)\right)+(z, \pi)\left(x, \frac{1}{\delta}(t-(1-\delta) T)\right) .
$$

Here, $w$ and $(z, \pi)$ are perturbations depending on $\alpha$ (which will be also fixed below), the initial state $y((1-\delta) T)$, and the desired state $y_{d}$. In order to drive (3.1) to a final state close to $y_{d}$, it is natural (at least formally) to look for a control close to $\widetilde{v}$ for $t \in[(1-\delta) T, T]$.

- We introduce a second control function by modifying $\widetilde{v}$ just as needed. Thus, we solve the following problem:

$$
\begin{cases}y_{t}+(y \cdot \nabla) y-\Delta y+\nabla p=v 1_{\mathcal{O}}+(\nabla \times \bar{y})(y-\widetilde{y})^{\perp}, \quad \nabla \cdot y=0 & \text { in } Q, \\ y \cdot n=0, \quad \nabla \times y=0 & \text { on } \Sigma, \\ y(0)=y^{0} & \text { in } \Omega .\end{cases}
$$


Here, we have introduced the function

$$
\bar{y}(x, t)=\frac{1}{\delta} y_{\alpha}\left(x, \frac{1}{\delta}(t-(1-\delta) T)\right), \quad\left(a_{1}, a_{2}\right)^{\perp}=\left(-a_{2}, a_{1}\right) .
$$

Since $\nabla \times \bar{y}$ vanishes outside $\mathcal{O} \times(0, T)$, it is clear that $(y, p)$ solves $(3.1)$ with

$$
v=\widetilde{v}+(\nabla \times \bar{y})(y-\widetilde{y})^{\perp} .
$$

Now, the task is reduced to show that for every $\varepsilon>0$, there exist positive $\alpha$ and $\delta$ such that

$$
\left\|y(T)-y_{d}\right\|_{W^{-1, \infty}} \leq \varepsilon .
$$

This can be achieved in the following way. Let us set $R=y-y^{0}, \omega=\nabla \times R$. Then

$$
\partial_{t} \omega+\left(R+y^{0}\right) \cdot \nabla \omega-\Delta \omega=-(R+z) \cdot \nabla(\nabla \times z)+\Delta(\nabla \times z)
$$

in $\Omega \times((1-\delta) T, T)$. Furthermore, $R(x,(1-\delta) T) \equiv 0$ and $z$ and all its derivatives of order $\leq 3$ are uniformly bounded. This leads, at first time, to a pointwise estimate of $\omega$ and then to an estimate of $R(\cdot, T)$ in $W^{-1, \infty}(\Omega)$ when $\alpha$ and $\delta$ are sufficiently small. Consequently, for any given $\varepsilon>0$, there exist $\alpha^{0}>0$ and $\eta:\left(0, \alpha^{0}\right) \mapsto \mathbf{R}_{+}$ such that whenever $0<\alpha<\alpha^{0}$ and $0<\delta<\eta(\alpha)$, one has (3.4) (for further details, see $[10])$.

This method has several limitations. Thus, the boundary conditions have to be of the Navier-slip type. In practice, this is equivalent to prescribing the values on the boundary of the stream function and the vorticity. At present, it is unknown how the method has to be modified in order to keep its validity in the context of Dirichlet (no-slip) boundary conditions. The case in which $\Omega$ is a manifold without boundary is considered in [11].

An extension of this method to Boussinesq systems in spatial dimensions $N=2$ and $N=3$ has been given in [31]. For the analysis of the similar three-dimensional situation for the Euler equations, see [33].

In the remainder of this section, we will be concerned with the null controllability of Stokes-like and Navier-Stokes systems completed with Dirichlet boundary conditions. Let us recall the definition of the following spaces, which are usual in the analysis of Stokes systems:

$$
\mathcal{V}=\left\{z \in C_{0}^{\infty}(\Omega)^{N}: \nabla \cdot z=0 \text { in } \Omega\right\}
$$

and

$$
H(\text { resp., } V)=\text { the adherence of } \mathcal{V} \text { in } L^{2}(\Omega)^{N}\left(\text { resp., } H_{0}^{1}(\Omega)^{N}\right) .
$$

We have

$$
H=\left\{z \in L^{2}(\Omega)^{N}: \nabla \cdot z=0 \text { in } \Omega, z \cdot n=0 \text { on } \partial \Omega\right\}
$$

and

$$
V=\left\{z \in H_{0}^{1}(\Omega)^{N}: \nabla \cdot z=0 \text { in } \Omega\right\} .
$$

For instance, see [55]. 
3.1. Null controllability of Stokes systems. We are first interested in proving the null controllability of some systems of the Stokes kind with distributed control. In other words, we would like to be able to find $v, y$, and $p$ such that

$$
\begin{cases}y_{t}-\Delta y+\nabla \cdot(y \otimes \bar{y})+\nabla \cdot(\bar{y} \otimes y)+\nabla p=v 1_{\mathcal{O}} & \text { in } Q, \\ \nabla \cdot y=0 & \text { in } Q, \\ y=0 & \text { on } \Sigma, \\ y(0)=y^{0} & \text { in } \Omega\end{cases}
$$

and

$$
y(T)=0 \quad \text { in } \Omega
$$

for any $y^{0} \in H$. Here, we will assume that the following regularity properties hold:

$$
\bar{y} \in L^{\infty}(Q)^{N}, \quad \bar{y}_{t} \in L^{2}\left(0, T ; L^{\sigma}(\Omega)\right)^{N},
$$

with $\sigma>1$ if $N=2$ and $\sigma>6 / 5$ if $N=3$.

Let us remark that (3.7) can be viewed as the linearized Navier-Stokes system, so we are actually trying to prove the exact controllability to the trajectories of those equations. In fact, this will serve to deduce the local exact controllability to the trajectories of the Navier-Stokes equations.

A previous result of this kind was proved in [37] under stronger hypotheses on the trajectory $\bar{y}$. In what follows, we will sketch the arguments presented in [24], which are also based on those in [37].

Concerning the null controllability of (3.7), we will first recall a Carleman inequality for the backwards system

$$
\begin{cases}-\varphi_{t}-\Delta \varphi-(D \varphi) \bar{y}+\nabla \pi=0 & \text { in } Q \\ \nabla \cdot \varphi=0 & \text { in } Q \\ \varphi=0 & \text { on } \Sigma \\ \varphi(T)=\varphi^{0} & \text { in } \Omega\end{cases}
$$

where $D \varphi=\nabla \varphi+\nabla \varphi^{t}$ and, consequently, the $i$ th component of $(D \varphi) \bar{y}$ is

$$
((D \varphi) \bar{y})_{i}=\sum_{j=1}^{N}\left(\partial_{j} \varphi^{i}+\partial_{i} \varphi^{j}\right) \bar{y}_{j} .
$$

To this end, let us introduce the following weight functions:

$$
\begin{aligned}
\beta(x, t) & =\frac{e^{5 / 4 \lambda k\|\eta\|_{\infty}}-e^{\lambda\left(k\|\eta\|_{\infty}+\eta(x)\right)}}{t^{4}(T-t)^{4}}, \quad \gamma(x, t)=\frac{e^{\lambda\left(k\|\eta\|_{\infty}+\eta(x)\right)}}{t^{4}(T-t)^{4}}, \\
\widehat{\beta}(t) & =\min _{x \in \bar{\Omega}} \beta(x, t), \quad \beta^{*}(t)=\max _{x \in \bar{\Omega}} \beta(x, t), \\
\widehat{\gamma}(t) & =\max _{x \in \bar{\Omega}} \gamma(x, t), \quad \gamma^{*}(t)=\min _{x \in \bar{\Omega}} \gamma(x, t) .
\end{aligned}
$$

Here, $k>4$ is a fixed number and $\eta$ is the function given by Lemma 1.2 associated to $\omega=\mathcal{O}_{5} \subset \subset \mathcal{O}$. Then we have the following. 
Lemma 3.1. Let us assume that (3.9) is satisfied. Then for every $\varphi^{0} \in H$ there exist three positive constants $\bar{s}, \bar{\lambda}$, and $C$ depending only on $\Omega$ and $\mathcal{O}$, such that

$$
\begin{aligned}
& s^{-1} \iint_{Q} e^{-2 s \beta} \gamma^{-1}|\varphi t|^{2} d x d t+s^{-1} \iint_{Q} e^{-2 s \beta} \gamma^{-1}|\Delta \varphi|^{2} d x d t \\
& +s \lambda^{2} \iint_{Q} e^{-2 s \beta} \gamma|\nabla \varphi|^{2} d x d t+s^{3} \lambda^{4} \iint_{Q} e^{-2 s \beta} \gamma^{3}|\varphi|^{2} d x d t \\
& \quad \leq C s^{16} \lambda^{40}\left(1+T^{2}\right) \iint_{\mathcal{O} \times(0, T)} e^{-8 s \widehat{\beta}+6 s \beta^{*}} \widehat{\gamma}^{16}|\varphi|^{2} d x d t
\end{aligned}
$$

for all $\lambda \geq \bar{\lambda}\left(1+\|\bar{y}\|_{\infty}+\left\|\bar{y}_{t}\right\|_{L^{2}\left(L^{\sigma}\right)}^{2}+e^{\left.\bar{\lambda} T\|\bar{y}\|_{\infty}^{2}\right)}\right.$ and $s \geq \bar{s}\left(T^{4}+T^{8}\right)$.

The proof of Lemma 3.1 is given in the next subsection. Notice that the hypotheses imposed above over $\bar{y}$ are not completely satisfactory. In fact, it is expected (and desirable) that an inequality like (3.13) hold for any $\bar{y} \in L^{\infty}(Q)^{N}$, but for the moment this is unknown.

Now, from this Carleman inequality, one can deduce the null controllability of (3.7) in a classical way.

THEOREM 3.2. Let us assume that (3.9) holds. Then for any $T>0$, the linear system (3.7) is null controllable with controls $v \in L^{2}(\mathcal{O} \times(0, T))^{N}$ at time $T$.

After some additional work, one can also deduce some local controllability results for the Navier-Stokes equations. Specifically, we mean local exact controllability to the trajectories. Thus, let us introduce the Navier-Stokes system

$$
\begin{cases}y_{t}-\Delta y+\nabla \cdot(y \otimes y)+\nabla p=v 1_{\mathcal{O}} & \text { in } Q, \\ \nabla \cdot y=0 & \text { in } Q, \\ y=0 & \text { on } \Sigma, \\ y(0)=y^{0} & \text { in } \Omega .\end{cases}
$$

We have the following.

TheOREM 3.3. Let $(\bar{y}, \bar{p})$ be a solution to the Navier-Stokes problem

$$
\begin{cases}\bar{y}_{t}-\Delta \bar{y}+\nabla \cdot(\bar{y} \otimes \bar{y})+\nabla \bar{p}=0 & \text { in } Q, \\ \nabla \cdot \bar{y}=0 & \text { in } Q, \\ \bar{y}=0 & \text { on } \Sigma, \\ \bar{y}(0)=\bar{y}^{0} & \text { in } \Omega\end{cases}
$$

verifying (3.9), and let us introduce the space E, with

$$
E=H \quad \text { if } N=2 \quad \text { and } \quad E=H \cap L^{4}(\Omega)^{3} \quad \text { if } N=3 .
$$

Then there exists $\varepsilon>0$ such that for each $y^{0} \in E$ satisfying $\left\|\bar{y}^{0}-y^{0}\right\|_{E} \leq \varepsilon$, there exist controls $v \in L^{2}(\mathcal{O} \times(0, T))^{N}$ and associated states $(y, p)$ such that one has (3.14) and

$$
y(T)=\bar{y}(T) \quad \text { in } \quad \Omega .
$$

This result can be proved by arguing as follows. We deduce in a first step a null controllability result for (3.7) with suitable right-hand side $f$. 
More precisely, let us set $L y=y_{t}-\Delta y+\nabla \cdot(\bar{y} \otimes y+y \otimes \bar{y})$ and let us introduce the spaces $E_{N}$, with

$$
\begin{aligned}
E_{2}=\{ & (y, v): e^{2 s \widehat{b}-s b^{*}} \widehat{\gamma}^{-15 / 4} y, e^{4 s \widehat{b}-3 s b^{*}} \widehat{\gamma}^{-8} v 1_{\omega} \in L^{2}(Q)^{2}, \\
& e^{s b^{*} / 2}\left(\gamma^{*}\right)^{-1 / 4} y \in L^{2}(0, T ; V) \cap L^{\infty}(0, T ; H), \\
& \left.\exists p: e^{s b^{*}}\left(\gamma^{*}\right)^{-1 / 2}\left(L y+\nabla p-v 1_{\omega}\right) \in L^{2}\left(0, T ; H^{-1}(\Omega)^{2}\right)\right\}
\end{aligned}
$$

and

$$
\begin{aligned}
E_{3}=\{ & (y, v): e^{2 s \widehat{b}-s b^{*}} \widehat{\gamma}^{-15 / 4} y, e^{4 s \widehat{b}-3 s b^{*}} \widehat{\gamma}^{-8} v 1_{\omega} \in L^{2}(Q)^{3}, \\
& e^{s b^{*} / 2}\left(\gamma^{*}\right)^{-1 / 4} y \in L^{2}(0, T ; V) \cap L^{\infty}(0, T ; H), \\
& e^{s b^{*} / 2}\left(\gamma^{*}\right)^{-1 / 4} y \in L^{4}\left(0, T ; L^{12}(\Omega)^{3}\right), \\
& \left.\exists p: e^{s b^{*}}\left(\gamma^{*}\right)^{-1 / 2}\left(L y+\nabla p-v 1_{\omega}\right) \in L^{2}\left(0, T ; W^{-1,6}(\Omega)^{3}\right)\right\},
\end{aligned}
$$

where the new weight functions $b, b^{*}$, etc., are given by

$$
\begin{aligned}
& b(x, t)=\frac{e^{5 / 4 \lambda m\left\|\eta^{0}\right\|_{\infty}}-e^{\lambda\left(m\left\|\eta^{0}\right\|_{\infty}+\eta^{0}(x)\right)}}{\ell(t)^{4}}, \\
& \widehat{b}(t)=\min _{x \in \bar{\Omega}} b(x, t), \quad b^{*}(t)=\max _{x \in \bar{\Omega}} b(x, t), \\
& \gamma(x, t)=\frac{e^{\lambda\left(m\left\|\eta^{0}\right\|_{\infty}+\eta^{0}(x)\right)}}{\ell(t)^{4}}, \\
& \widehat{\gamma}(t)=\max _{x \in \bar{\Omega}} \gamma(x, t), \quad \gamma^{*}(t)=\min _{x \in \bar{\Omega}} \gamma(x, t) .
\end{aligned}
$$

Here, we have introduced

$$
\ell(t)=\left\{\begin{array}{lll}
T^{2} / 4 & \text { for } & 0<t<T / 2, \\
t(T-t) & \text { for } & T / 2<t<T .
\end{array}\right.
$$

We then have the following.

Proposition 3.4. Assume that $\bar{y}$ satisfies (3.9) and

- $y^{0} \in H, e^{s b^{*}}\left(\gamma^{*}\right)^{-1 / 2} f \in L^{2}\left(0, T ; H^{-1}(\Omega)^{2}\right)$ if $N=2$;

- $y^{0} \in L^{4}(\Omega)^{3} \cap H, e^{s b^{*}}\left(\gamma^{*}\right)^{-1 / 2} f \in L^{2}\left(0, T ; W^{-1,6}(\Omega)^{3}\right)$ if $N=3$.

Then there exists a control $v \in L^{2}(\omega \times(0, T))^{N}$ such that if $y$ is the associated solution to (3.7), we have $(y, v) \in E_{N}$.

Notice that this is actually a null controllability result for (3.7). Indeed, if $(y, v) \in$ $E_{N}$, we have in particular that $y(T)=0$ in $\Omega$.

The rest of the proof of Theorem 3.3 relies on an appropriate inverse mapping theorem. More precisely, we use the following result from [1].

Proposition 3.5. Let $X, F$ be two Banach spaces and let $\mathcal{A}: X \mapsto F$ satisfy $\mathcal{A} \in$ $C^{1}(X ; F)$. Assume that $e_{0} \in X, \mathcal{A}\left(e_{0}\right)=h_{0}$, and $\mathcal{A}^{\prime}\left(e_{0}\right): X \mapsto F$ is an epimorphism. Then there exists $\delta>0$ such that for every $h \in F$ satisfying $\left\|h-h_{0}\right\|_{F}<\delta$, there exists a solution of the equation

$$
\mathcal{A}(e)=h, \quad e \in X
$$

Let us consider the mapping $\mathcal{A}: X \mapsto F$, given by

$$
\mathcal{A}(y, v)=\left(L y+(y \cdot \nabla) y+\nabla p-v 1_{\omega}, y(\cdot, 0)\right) \quad \forall(y, v) \in X,
$$


where $X=E_{N}$ and

$$
F= \begin{cases}L^{2}\left(e^{s b^{*}}\left(\gamma^{*}\right)^{-1 / 2} ; H^{-1}(\Omega)^{2}\right) \times H & \text { if } N=2, \\ L^{2}\left(e^{s b^{*}}\left(\gamma^{*}\right)^{-1 / 2} ; W^{-1,6}(\Omega)^{3}\right) \times\left(L^{4}(\Omega)^{3} \cap H\right) & \text { if } N=3 .\end{cases}
$$

From the definition of $E_{N}$, one can easily check that $\mathcal{A}$ is well defined and satisfies $\mathcal{A} \in C^{1}(X ; F)$. Furthermore, the identity

$$
\operatorname{Im}\left(\mathcal{A}^{\prime}(0,0)\right)=F
$$

is equivalent to the result stated in Proposition 3.4. Therefore, we can apply Proposition 3.5 to $\mathcal{A}$ with $e_{0}=(0,0)$ and $h_{0}=(0,0)$. This ends the proof of Theorem 3.3.

For more details concerning the local exact controllability of (3.14), see [24]; see also [25].

3.2. Proof of the Carleman inequality. In this subsection, we sketch the proof of Lemma 3.1. This is contained in a more general case covered by the results in [24]. Throughout the proof, $C$ will denote a generic positive constant that may depend on $\Omega$ and $\mathcal{O}$ and can change its value from one line to the next.

For easier comprehension, we will divide the proof in several steps.

3.2.1. Carleman estimate for the heat equation and first estimate of the pressure. Let us apply the Carleman inequality (1.18) to each component $\varphi^{i}$ with right-hand side

$$
-\partial_{i} \pi+((D \varphi) \bar{y})_{i} \quad(1 \leq i \leq N) .
$$

Introducing the notation

$$
\begin{aligned}
I(s, \lambda ; \varphi)= & s^{-1} \iint_{Q} e^{-2 s b} \gamma^{-1}\left(\left|\varphi_{t}\right|^{2}+|\Delta \varphi|^{2}\right) d x d t \\
& +s \lambda^{2} \iint_{Q} e^{-2 s \beta} \gamma|\nabla \varphi|^{2} d x d t+s^{3} \lambda^{4} \iint_{Q} e^{-2 s \beta} \gamma^{3}|\varphi|^{2} d x d t,
\end{aligned}
$$

one has

$$
\begin{aligned}
I(s, \lambda ; \varphi) \leq C & \left(s^{3} \lambda^{4} \iint_{\mathcal{O}_{5} \times(0, T)} e^{-2 s \beta} \gamma^{3}|\varphi|^{2} d x d t\right. \\
& \left.+\iint_{Q} e^{-2 s \beta}\left(|\nabla \pi|^{2}+|(D \varphi) \bar{y}|^{2}\right) d x d t\right)
\end{aligned}
$$

for $\lambda \geq C$ and $s \geq C\left(T^{7}+T^{8}\right)$.

Taking $\lambda \geq \bar{C}\|\bar{y}\|_{\infty}$ and $s \geq C T^{8}$, one can eliminate the term $|(D \varphi) \bar{y}|^{2}$ on the right-hand side, whence we obtain

$$
I(s, \lambda ; \varphi) \leq C\left(s^{3} \lambda^{4} \iint_{\mathcal{O}_{5} \times(0, T)} e^{-2 s \beta} \gamma^{3}|\varphi|^{2} d x d t+\iint_{Q} e^{-2 s \beta}|\nabla \pi|^{2} d x d t\right)
$$

for $\lambda \geq C\left(1+\|\bar{y}\|_{\infty}\right)$ and $s \geq C\left(T^{4}+T^{8}\right)$.

Let us now present an estimate of the last integral. To this end, we will apply the main result in [38] to the elliptic equation

$$
-\Delta \pi(t)=-\nabla \cdot((D \varphi) \bar{y})(t) \quad \text { in } \quad \Omega
$$


for each $t \in(0, T)$. This provides

$$
\begin{aligned}
\int_{\Omega} e^{2 \tau e^{\lambda \eta}}|\nabla \pi(t)|^{2} d x \leq & C\left(\int_{\mathcal{O}_{5}} e^{2 \tau e^{\lambda \eta}}\left(|\nabla \pi(t)|^{2}+\tau^{2} \lambda^{2} e^{2 \lambda \eta}|\pi(t)|^{2}\right) d x\right. \\
& \left.+\tau \int_{\Omega} e^{2 \tau e^{\lambda \eta}} e^{\lambda \eta}|(D \varphi) \bar{y}(t)|^{2} d x+\tau^{1 / 2} e^{2 \tau}\|\pi(t)\|_{H^{1 / 2}(\partial \Omega)}^{2}\right)
\end{aligned}
$$

for $\lambda, \tau \geq C$.

One can readily see that the local term of $|\nabla \pi|^{2}$ can be eliminated by arguing as in the proof of Lemma 1.3, so we obtain

$$
\begin{aligned}
\int_{\Omega} e^{2 \tau e^{\lambda \eta}}|\nabla \pi(t)|^{2} d x \leq & C\left(\tau^{2} \lambda^{2} \int_{\mathcal{O}_{4}} e^{2 \tau e^{\lambda \eta}} e^{2 \lambda \eta}|\pi(t)|^{2} d x\right. \\
& \left.+\tau \int_{\Omega} e^{2 \tau e^{\lambda \eta}} e^{\lambda \eta}|(D \varphi) \bar{y}(t)|^{2} d x+\tau^{1 / 2} e^{2 \tau}\|\pi(t)\|_{H^{1 / 2}(\partial \Omega)}^{2}\right)
\end{aligned}
$$

for an open subset $\mathcal{O}_{4}$ verifying $\mathcal{O}_{5} \subset \subset \mathcal{O}_{4} \subset \subset \mathcal{O}$.

Next, we put

$$
\tau=\frac{s}{t^{4}(T-t)^{4}} e^{\lambda k\|\eta\|_{\infty}}
$$

we multiply (3.24) by

$$
\exp \left\{-2 s \frac{e^{5 / 4 \lambda k\|\eta\|_{\infty}}}{t^{4}(T-t)^{4}}\right\}
$$

and we integrate with respect to $t$ in $(0, T)$. This yields

$$
\begin{aligned}
\iint_{Q} e^{-2 s \beta}|\nabla \pi|^{2} d x d t \leq C & \left(s^{2} \lambda^{2} \iint_{\mathcal{O}_{4} \times(0, T)} e^{-2 s \beta} \gamma^{2}|\pi|^{2} d x d t\right. \\
& +s \iint_{Q} e^{-2 s \beta} \gamma|(D \varphi) \bar{y}|^{2} d x d t \\
& \left.+s^{1 / 2} \int_{0}^{T} e^{-2 s \beta^{*}}\left(\gamma^{*}\right)^{1 / 2}\|\pi(t)\|_{H^{1 / 2}(\partial \Omega)}^{2} d t\right)
\end{aligned}
$$

for $\lambda \geq C$ and $s \geq C T^{8}$.

Let us now estimate the term containing the trace of $\pi$ following the ideas of [37]. Thus, let us introduce the functions

$$
\varphi^{*}=s^{1 / 4} e^{-s \beta^{*}}\left(\gamma^{*}\right)^{1 / 4} \varphi \quad \text { and } \quad \pi^{*}=s^{1 / 4} e^{-s \beta^{*}}\left(\gamma^{*}\right)^{1 / 4} \pi,
$$

which satisfy

$$
\begin{cases}-\varphi_{t}^{*}-\Delta \varphi^{*}+\nabla \pi^{*}=\left(D \varphi^{*}\right) \bar{y}-s^{1 / 4}\left(e^{-s \beta^{*}}\left(\gamma^{*}\right)^{1 / 4}\right)_{t} \varphi & \text { in } Q \\ \nabla \cdot \varphi^{*}=0 & \text { in } Q \\ \varphi^{*}=0 & \text { on } \Sigma, \\ \varphi^{*}(T)=0 & \text { in } \Omega .\end{cases}
$$


Using well-known regularity properties of the Stokes system, we find

$$
\begin{aligned}
\left\|\pi^{*}\right\|_{L^{2}\left(0, T ; H^{1 / 2}(\partial \Omega)\right)}^{2} \leq & C\left(\iint_{Q}\left(\left|D \varphi^{*} \bar{y}\right|^{2}+s^{1 / 2}\left|\left(e^{-s \beta^{*}}\left(\gamma^{*}\right)^{1 / 4}\right)\right|_{t}^{2}|\varphi|^{2}\right) d x d t\right) \\
\leq & C\left(s^{1 / 2} \iint_{Q} e^{-2 s \beta^{*}}\left(\gamma^{*}\right)^{1 / 2}|(D \varphi) \bar{y}|^{2} d x d t\right. \\
& \left.+s^{5 / 2} T^{2} \iint_{Q} e^{-2 s \beta^{*}}\left(\gamma^{*}\right)^{3}|\varphi|^{2} d x d t\right)
\end{aligned}
$$

for $s \geq C T^{8}$. This, used in combination with (3.27), leads to the inequality

$$
\begin{aligned}
\iint_{Q} e^{-2 s \beta}|\nabla \pi|^{2} d x d t \leq C & \left(s^{2} \lambda^{2} \iint_{\mathcal{O}_{4} \times(0, T)} e^{-2 s \beta} \gamma^{2}|\pi|^{2} d x d t\right. \\
& +s \iint_{Q} e^{-2 s \beta} \gamma|(D \varphi) \bar{y}|^{2} d x d t \\
& \left.+s^{5 / 2} T^{2} \iint_{Q} e^{-2 s \beta} \gamma^{3}|\varphi|^{2} d x d t\right),
\end{aligned}
$$

which must hold for $\lambda \geq C$ and $s \geq C T^{8}$.

We finally insert this inequality into (3.21) and absorb the last two terms on the right-hand side:

$$
I(s, \lambda ; \varphi) \leq C s^{2} \lambda^{2} \iint_{\mathcal{O}_{4} \times(0, T)} e^{-2 s \beta}\left(s \lambda^{2} \gamma^{3}|\varphi|^{2}+\gamma^{2}|\pi|^{2}\right) d x d t
$$

for $\lambda \geq C\left(1+\|\bar{y}\|_{\infty}\right)$ and $s \geq C\left(T^{4}+T^{8}\right)$.

3.2.2. Local estimate of the pressure. The goal of this paragraph is to estimate the local integral of $\pi$ appearing in (3.32). This is the most complicated part of the proof. Let us assume that we have chosen $\pi$ such that

$$
\int_{\mathcal{O}_{4}} \pi(t) d x=0 \quad \forall t \in(0, T) .
$$

Then from the definition of the weight functions and the Poincaré-Wirtinger inequality, we have

$$
\begin{aligned}
s^{2} \lambda^{2} \iint_{\mathcal{O}_{4} \times(0, T)} e^{-2 s \beta} \gamma^{2}|\pi|^{2} d x d t & \leq s^{2} \lambda^{2} \iint_{\mathcal{O}_{4} \times(0, T)} e^{-2 s \widehat{\beta}} \widehat{\gamma}^{2}|\pi|^{2} d x d t \\
& \leq C s^{2} \lambda^{2} \iint_{\mathcal{O}_{4} \times(0, T)} e^{-2 s \widehat{\beta}} \widehat{\gamma}^{2}|\nabla \pi|^{2} d x d t .
\end{aligned}
$$

Recall that $\widehat{\beta}=\widehat{\beta}(t)$ and $\widehat{\gamma}=\widehat{\gamma}(t)$ are given as follows:

$$
\widehat{\beta}(t)=\min _{\bar{\Omega}} \beta(x, t), \quad \widehat{\gamma}(t)=\max _{\bar{\Omega}} \gamma(x, t) .
$$

From the differential equation in (3.10), we deduce that in order to estimate the last integral in (3.34), it suffices to bound locally the Laplacian and the time derivative of the velocity field $\varphi$. 
A local estimate of $\Delta \varphi$. Let us first introduce two open sets $\mathcal{O}_{2}$ and $\mathcal{O}_{3}$, with

$$
\mathcal{O}_{4} \subset \subset \mathcal{O}_{3} \subset \subset \mathcal{O}_{2} \subset \subset \mathcal{O},
$$

and a function $\rho \in \mathcal{D}\left(\mathcal{O}_{2}\right)$, with $\rho \equiv 1$ in $\mathcal{O}_{3}$.

Next, we put

$$
u(x, t)=\widehat{p}(t) \rho(x) \Delta \varphi(x, T-t) \quad \forall(x, t) \in \mathbf{R}^{N} \times(0, T),
$$

where we have denoted by $\widehat{p}$ the function $\widehat{p}(t)=s \lambda e^{-s \widehat{\beta}} \widehat{\gamma}$.

From (3.10), we obtain

$$
(\Delta \varphi(T-t))_{t}-\Delta(\Delta \varphi(T-t))=f \quad \text { in } Q
$$

with

$$
f(t)=\Delta((D \varphi) \bar{y})(T-t)-\nabla(\nabla \cdot((D \varphi) \bar{y})(T-t)) .
$$

This provides the following heat problem for the function $u$ :

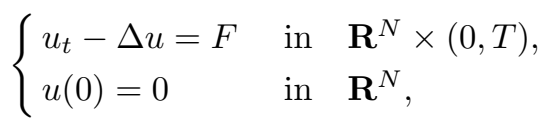

with

$$
F(t)=\widehat{p} \rho f+\widehat{p}^{\prime} \rho \Delta \varphi(T-t)-2 \widehat{p} \nabla \rho \cdot \nabla \Delta \varphi(T-t)-\widehat{p} \Delta \rho \Delta \varphi(T-t)
$$

for all $t \in(0, T)$. Then we rewrite $F$ in the form $F=F_{1}+F_{2}$, with

$$
\begin{aligned}
F_{1}(t)= & \widehat{p} \Delta(\rho((D \varphi) \bar{y})(T-t))-\widehat{p} \nabla(\nabla \cdot(\rho((D \varphi) \bar{y})(T-t))) \\
& +\widehat{p}^{\prime} \Delta(\rho \varphi(T-t))
\end{aligned}
$$

and

$$
\begin{aligned}
F_{2}(t)= & -2 \widehat{p} \nabla \rho \cdot \nabla((D \varphi) \bar{y})(T-t)-\widehat{p} \Delta \rho((D \varphi) \bar{y})(T-t) \\
& +\widehat{p} \nabla(\nabla \rho \cdot((D \varphi) \bar{y})(T-t))+\widehat{p} \nabla \rho \cdot(\nabla \cdot((D \varphi) \bar{y})(T-t)) \\
& -2 \widehat{p}^{\prime} \nabla \rho \cdot \nabla \varphi(T-t)-\widehat{p}^{\prime} \Delta \rho \varphi(T-t)-2 \widehat{p} \nabla \rho \cdot \nabla \Delta \varphi(T-t) \\
& -\widehat{p} \Delta \rho \Delta \varphi(T-t),
\end{aligned}
$$

and we define $u^{i}(i=1,2)$ as the solution to

$$
\left\{\begin{array}{lll}
u_{t}^{i}-\Delta u^{i}=F_{i} & \text { in } & \mathbf{R}^{N} \times(0, T), \\
u^{i}(0)=0 & \text { in } & \mathbf{R}^{N} .
\end{array}\right.
$$

An estimate of $u^{1}$. Let us remark that $F_{1} \in L^{2}\left(0, T ; H^{-2}\left(\mathbf{R}^{N}\right)^{N}\right)$, while we would like to estimate the $L^{2}$-norm of $u^{1}$. Accordingly, we have to define $u^{1}$ like a solution by transposition. More precisely, $u^{1}$ is the unique function in $L^{2}\left(\mathbf{R}^{N} \times(0, T)\right)^{N}$ verifying

$$
\begin{aligned}
\iint_{\mathbf{R}^{N} \times(0, T)} u^{1} \cdot k d x d t= & \iint_{\mathbf{R}^{N} \times(0, T)} \widehat{p} \rho((D \varphi) \bar{y}(T-t)) \cdot \Delta z d x d t \\
& -\iint_{\mathbf{R}^{N} \times(0, T)} \widehat{p} \rho((D \varphi) \bar{y}(T-t)) \cdot \nabla(\nabla \cdot z) d x d t \\
& +\iint_{\mathbf{R}^{N} \times(0, T)} \widehat{p}^{\prime} \rho \varphi(T-t) \cdot \Delta z d x d t
\end{aligned}
$$


for any $k \in L^{2}\left(\mathbf{R}^{N} \times(0, T)\right)^{N}$, where $z$ is the solution of

$$
\left\{\begin{array}{lll}
-z_{t}-\Delta z=k & \text { in } & \mathbf{R}^{N} \times(0, T), \\
z(T)=0 & \text { in } & \mathbf{R}^{N}
\end{array}\right.
$$

Then we get the following directly from (3.45):

$$
\begin{aligned}
& \iint_{\mathbf{R}^{N} \times(0, T)}\left|u^{1}\right|^{2} d x d t \\
& \quad \leq C\left(\iint_{\mathbf{R}^{N} \times(0, T)}|\widehat{p} \rho(D \varphi) \bar{y}|^{2} d x d t+\iint_{\mathbf{R}^{N} \times(0, T)}\left|\widehat{p}^{\prime} \rho \varphi\right|^{2} d x d t\right) \\
& \quad \leq C\left(\left.\iint_{\mathcal{O}_{2} \times(0, T)}\left|\widehat{p}^{2}\right|(D \varphi) \bar{y}\right|^{2} d x d t+\iint_{\mathcal{O}_{2} \times(0, T)}\left|\widehat{p}^{\prime}\right|^{2}|\varphi|^{2} d x d t\right) .
\end{aligned}
$$

An estimate of $u^{2}$. This time we must take into account the fact that the function $F_{2}$ belongs to the space $L^{2}\left(0, T ; H^{-1}\left(\mathbf{R}^{N}\right)^{N}\right)$. But it is also essential to remember that supp $F_{2} \subset \mathcal{O}_{2} \backslash \overline{\mathcal{O}}_{3}$, since derivatives of $\rho$ appear everywhere. This allows us to write, for $x \in \mathcal{O}_{4}$,

$$
u^{2}(x, t)=\int_{0}^{t} \int_{\mathcal{O}_{2} \backslash \overline{\mathcal{O}}_{3}} G(x-y, t-s) F_{2}(y, s) d y d s,
$$

where $G$ is the fundamental solution of the heat equation, i.e.,

$$
G(x, t)=\frac{1}{(4 \pi t)^{N / 2}} e^{-\frac{|x|^{2}}{2 t}} \quad \forall(x, t) \in \mathbf{R}^{N} \times \mathbf{R}_{+} .
$$

Now, we integrate by parts with respect to $y$ in (3.48), taking all the space derivatives out of $(D \varphi) \bar{y}$ and $\varphi$. This gives the following equality:

$$
u^{2}(x, t)=\int_{0}^{t} \int_{\mathcal{O}_{2} \backslash \overline{\mathcal{O}}_{3}} \sum_{\alpha \in I, \beta \in J} D_{y}^{\alpha} G(x-y, t-s) D_{y}^{\beta} \rho(y) w_{\alpha, \beta}(y, s) d y d s,
$$

where

$$
w_{\alpha, \beta}(y, s)=\left(A_{\alpha, \beta} \widehat{p}(s)+B_{\alpha, \beta} \widehat{p}^{\prime}(s)\right) \varphi(y, s)+C_{\alpha, \beta} \widehat{p}(s)((D \varphi) \bar{y})(y, s)
$$

for appropriate constants $A_{\alpha, \beta}, B_{\alpha, \beta}$, and $C_{\alpha, \beta}$,

$$
I \subset\left\{\gamma \in \mathrm{N}^{N}:|\gamma| \leq 3\right\} \quad \text { and } \quad J \subset\left\{\gamma \in \mathrm{N}^{N}: 1 \leq|\gamma| \leq 4\right\} .
$$

Next, we denote by $d$ the quantity $d=\operatorname{dist}\left(\partial \mathcal{O}_{3}, \partial \mathcal{O}_{4}\right)$, we fix $\delta \in(0, d)$, and we observe that

$$
\left|D_{y}^{\alpha} G(x-y, t-s)\right| \leq C \exp \left(-\frac{\delta^{2}}{2(t-s)}\right) \quad \forall x \in \mathcal{O}_{4}, \forall y \in \mathcal{O}_{2} \backslash \overline{\mathcal{O}}_{3} .
$$

Therefore, we deduce from (3.50) that

$$
\left|u^{2}(x, t)\right| \leq C \int_{0}^{t} \int_{\mathcal{O}_{2} \backslash \overline{\mathcal{O}}_{3}} e^{-\frac{\delta^{2}}{2(t-s)}}|w(y, s)| d y d s \quad \forall(x, t) \in \mathcal{O}_{4} \times(0, T),
$$


where

$$
w(y, s)=\left(\widehat{p}(s)+\widehat{p}^{\prime}(s)\right) \varphi(y, s)+\widehat{p}(s)((D \varphi) \bar{y})(y, s) .
$$

Integrating with respect to $x$ and $t$ in $\mathcal{O}_{4} \times(0, T)$, we find that

$$
\begin{aligned}
\iint_{\mathcal{O}_{4} \times(0, T)}\left|u^{2}\right|^{2} d x d t & \leq C T \int_{0}^{T} \int_{0}^{t} e^{-\delta^{2} /(t-s)}\|w(y, s)\|_{L^{2}\left(\mathcal{O}_{2}\right)}^{2} d s d t \\
& =C T \int_{0}^{T}\left(f_{1} * f_{2}\right)(t) d t
\end{aligned}
$$

where

$$
f_{1}(t)=e^{-\delta^{2} / t} \quad \text { and } \quad f_{2}(t)=\|w(t)\|_{L^{2}\left(\mathcal{O}_{2}\right)}^{2} .
$$

Notice that $f_{1}, f_{2} \in L^{1}(0, T)$. As a consequence of Young's inequality, we have

$$
\begin{aligned}
\iint_{\mathcal{O}_{4} \times(0, T)}\left|u^{2}\right|^{2} d x d t & \leq C T \iint_{\mathcal{O}_{2} \times(0, T)}|w|^{2} d x d t \\
\leq C T & \left(\iint_{\mathcal{O}_{2} \times(0, T)}\left(|\widehat{p}|^{2}+\left|\widehat{p}^{\prime}\right|^{2}\right)|\varphi|^{2} d x d t\right. \\
& \left.+\iint_{\mathcal{O}_{2} \times(0, T)}|\widehat{p}|^{2}|(D \varphi) \bar{y}|^{2} d x d t\right) .
\end{aligned}
$$

Combining this with (3.47), we obtain the local estimate of $\Delta \varphi$ :

$$
\begin{aligned}
s^{2} \lambda^{2} \iint_{\mathcal{O}_{4} \times(0, T)} e^{-2 s \widehat{\beta}} \widehat{\gamma}^{2}|\Delta \varphi|^{2} d x d t \leq C(1+T)( & \iint_{\mathcal{O}_{2} \times(0, T)}\left(|\widehat{p}|^{2}+\left|\widehat{p}^{2}\right|^{2}\right)|\varphi|^{2} d x d t \\
& \left.+\iint_{\mathcal{O}_{2} \times(0, T)}|\widehat{p}|^{2}|(D \varphi) \bar{y}|^{2} d x d t\right) .
\end{aligned}
$$

A local estimate of $\varphi_{t}$. Let us first introduce the weight function $p$, with

$$
p(t)=s^{15 / 4} e^{-2 s \widehat{\beta}+s \beta^{*}} \widehat{\gamma}^{15 / 4} .
$$

By setting $(\psi, \theta)=(p \varphi, p \pi)$, we have

$$
\begin{cases}-\psi_{t}-\Delta \psi-(D \psi) \bar{y}+\nabla \theta=-p^{\prime} \varphi & \text { in } Q, \\ \nabla \cdot \psi=0 & \text { in } Q, \\ \psi=0 & \text { on } \Sigma, \\ \psi(T)=0 & \text { in } \Omega .\end{cases}
$$

Recall that the term we have to estimate is

$$
\begin{aligned}
\iint_{\mathcal{O}_{4} \times(0, T)}|\widehat{p}|^{2}\left|\varphi_{t}\right|^{2} d x d t & =\iint_{\mathcal{O}_{4} \times(0, T)} p^{-2}|\widehat{p}|^{2}\left|p \varphi_{t}\right|^{2} d x d t \\
& =s^{-11 / 2} \lambda^{2} \iint_{\mathcal{O}_{4} \times(0, T)} e^{-2 s \beta^{*}+2 s \widehat{\beta}} \widehat{\gamma}^{-11 / 2}\left|\psi_{t}-p^{\prime} \varphi\right|^{2} d x d t .
\end{aligned}
$$


Hence, we will try to estimate the (weighted) $L^{2}$-norm of the time derivative of $\psi$. To this end, we integrate by parts with respect to $t$ :

$$
\begin{aligned}
& s^{-11 / 2} \lambda^{2} \iint_{\mathcal{O}_{4} \times(0, T)} e^{-2 s \beta^{*}+2 s \widehat{\beta}} \widehat{\gamma}^{-11 / 2}\left|\psi_{t}\right|^{2} d x d t \\
& =\frac{1}{2} s^{-11 / 2} \lambda^{2} \iint_{\mathcal{O}_{4} \times(0, T)}\left(e^{-2 s \beta^{*}+2 s \widehat{\beta}} \widehat{\gamma}^{-11 / 2}\right)_{t t}|\psi|^{2} d x d t \\
& \quad-s^{-11 / 2} \lambda^{2} \iint_{\mathcal{O}_{4} \times(0, T)} e^{-2 s \beta^{*}+2 s \widehat{\beta}} \widehat{\gamma}^{-11 / 2} \psi_{t t} \psi d x d t .
\end{aligned}
$$

One can easily check that

$$
s^{-11 / 2} \lambda^{2}\left(e^{-2 s \beta^{*}+2 s \widehat{\beta}} \widehat{\gamma}^{-11 / 2}\right)_{t t} \leq C s^{-7 / 2} \lambda^{2} T^{2} e^{-2 s \beta^{*}+2 s \widehat{\beta}} \widehat{\gamma}^{-3}
$$

for $s \geq C T^{8}$.

Therefore, we must concentrate on estimating the last term in (3.63). Now, we first denote by $p^{*}$ the function

$$
p^{*}=s^{-11 / 2} \lambda^{-4} e^{-2 s \beta^{*}+2 s \widehat{\beta}} \widehat{\gamma}^{-11 / 2}
$$

and then use Hölder's inequality:

$$
\begin{aligned}
\lambda^{6} \iint_{\mathcal{O}_{4} \times(0, T)} p^{*} \psi_{t t} \psi d x d t \leq & \lambda^{6}\left\|p^{*} \psi_{t t}\right\|_{L^{2}\left(0, T ; L^{q}\left(\mathcal{O}_{4}\right)\right)}\|\psi\|_{L^{2}\left(0, T ; L^{q^{\prime}}\left(\mathcal{O}_{4}\right)\right)} \\
\leq & 2\left\|p^{*} \psi_{t t}\right\|_{L^{2}\left(0, T ; L^{q}\left(\mathcal{O}_{4}\right)\right)}^{2} \\
& +2 \lambda^{12}\|\psi\|_{L^{2}\left(0, T ; L^{q^{\prime}}\left(\mathcal{O}_{4}\right)\right)}^{2},
\end{aligned}
$$

where $q$ is chosen lower than $\sigma$ but greater than 1 in dimension $N=2$ and greater than $6 / 5$ in dimension $N=3$, and $q^{\prime}$ is the conjugate exponent of $q$ (recall that $\sigma>1$ if $N=2$ and $\sigma>6 / 5$ if $N=3$ ).

Let us first deal with the second term on the right-hand side of (3.66). Let $\rho \in C^{2}\left(\mathcal{O}_{3}\right)$ be a cut-off function satisfying

$$
\operatorname{supp} \rho \subset \mathcal{O}_{3} \quad \text { and } \quad \rho \equiv 1 \text { in } \mathcal{O}_{4} .
$$

Then

$$
\begin{aligned}
\|\psi\|_{L^{2}\left(L^{q^{\prime}}\left(\mathcal{O}_{4}\right)\right)}^{2} & \leq\|\Delta(\rho \psi)\|_{L^{2}\left(L^{2}\left(\mathcal{O}_{3}\right)\right)}^{2} \\
& =\|\Delta \rho \psi+2 \nabla \rho \cdot \nabla \psi+\rho \Delta \psi\|_{L^{2}\left(L^{2}\left(\mathcal{O}_{3}\right)\right)}^{2} .
\end{aligned}
$$

Following the same steps as in the previous section for obtaining the local estimate of $\Delta \varphi$, after some long computations, one can readily get an estimate of the last term on the right-hand side of (3.68). Consequently, we have

$$
\|\psi\|_{L^{2}\left(0, T ; L^{q^{\prime}}\left(\mathcal{O}_{4}\right)\right)}^{2} \leq C\left(1+\|\bar{y}\|_{\infty}^{2}\right)(1+T) \iint_{\mathcal{O}_{1} \times(0, T)}\left(|\psi|^{2}+|\nabla \psi|^{2}+\left|p^{\prime} \varphi\right|^{2}\right) d x d t,
$$

where $\mathcal{O}_{1}$ is an open set verifying

$$
\mathcal{O}_{2} \subset \subset \mathcal{O}_{1} \subset \subset \mathcal{O} \text {. }
$$


Finally, we treat the norm of $p^{*} \psi_{t t}$ in (3.66).

Observing the problem verified by $\left(p^{*} \psi_{t}, p^{*} \theta_{t}\right)$, one can obtain the next a priori estimate for $\psi_{t t}$ :

$$
\begin{aligned}
& \left\|p^{*} \psi_{t t}\right\|_{L^{2}\left(L^{q}\right)} \leq\left(1+\|\bar{y}\|_{\infty}\right) e^{C\left(1+T\|\bar{y}\|_{\infty}^{2}\right)}\left(\left\|p^{*} p^{\prime \prime} \varphi\right\|_{L^{2}\left(L^{2}\right)}\right. \\
& \left.+\left\|p^{*} p^{\prime} \varphi_{t}\right\|_{L^{2}\left(L^{2}\right)}+\left\|\left(p^{*}\right)^{\prime} \psi_{t}\right\|_{L^{2}\left(L^{2}\right)}+\left\|p^{*}(D \psi) \bar{y}_{t}\right\|_{L^{2}\left(L^{q}\right)}\right) .
\end{aligned}
$$

Consequently, from (3.62)-(3.66) and (3.69)-(3.71), we have

$$
\begin{aligned}
& \iint_{\mathcal{O}_{4} \times(0, T)}|\widehat{p}|^{2}\left|\varphi_{t}\right|^{2} d x d t \\
& \quad \leq\left(1+\|\bar{y}\|_{\infty}^{2}\right) e^{C\left(1+T\|\bar{y}\|_{\infty}^{2}\right)}\left(\lambda ^ { 1 2 } ( 1 + T ) \left(\|p \varphi\|_{L^{2}\left(L^{2}\left(\mathcal{O}_{1}\right)\right)}^{2}\right.\right. \\
& \left.\quad+\|p \nabla \varphi\|_{L^{2}\left(L^{2}\left(\mathcal{O}_{1}\right)\right)}^{2}+\left\|p^{\prime} \varphi\right\|_{L^{2}\left(L^{2}\left(\mathcal{O}_{1}\right)\right)}^{2}\right)+\left\|p^{*} p^{\prime \prime} \varphi\right\|_{L^{2}\left(L^{2}\right)}^{2} \\
& \left.\quad+\left\|p^{*} p^{\prime} \varphi_{t}\right\|_{L^{2}\left(L^{2}\right)}^{2}+\left\|\left(p^{*}\right)^{\prime} \psi_{t}\right\|_{L^{2}\left(L^{2}\right)}^{2}+\left\|p^{*}(D \psi) \bar{y}_{t}\right\|_{L^{2}\left(L^{q}\right)}^{2}\right) .
\end{aligned}
$$

An estimate of $\boldsymbol{p}^{*}(\boldsymbol{D} \psi) \overline{\boldsymbol{y}}_{\boldsymbol{t}}$. Let $\ell \in(1,+\infty)$ be given. In this section, we will prove that for $\bar{y} \in L^{\infty}(Q)^{N}$, we have $p^{*} \psi \in L^{\infty}\left(0, T ; W^{1, \ell}(\Omega)\right)^{N}$ for all $\ell<\infty$, and we will get an estimate of its norm. Once this is established, we will directly obtain an estimate of $p^{*}(D \psi) \bar{y}_{t}$ in $L^{2}\left(L^{q}\right)$ (taking $\ell$ large enough).

Thus, let us look at the system fulfilled by $\left(p^{*} \psi, p^{*} \theta\right)$ :

$$
\begin{cases}-\left(p^{*} \psi\right)_{t}-\Delta\left(p^{*} \psi\right)-D\left(p^{*} \psi\right) \bar{y}+\nabla\left(p^{*} \theta\right)=-\left(p^{*}\right)^{\prime} \psi-p^{*} p^{\prime} \varphi & \text { in } Q \\ \nabla \cdot\left(p^{*} \psi\right)=0 & \text { in } Q \\ p^{*} \psi=0 & \text { on } \Sigma \\ \left(p^{*} \psi\right)(T)=0 & \text { in } \Omega\end{cases}
$$

We can first view $\psi$ as a function in

$$
L^{2}\left(0, T ; H^{2}(\Omega)^{N}\right) \cap L^{\infty}(0, T ; V) .
$$

Then, using interpolation inequalities, we have that $D\left(p^{*} \psi\right) \bar{y} \in L^{k_{1}}\left(L^{k_{2}}\right)$, with $2<$ $k_{1}<4$ and $3<k_{2}<6$ (which will be chosen below) verifying

$$
\frac{4 / 3}{k_{1}}+\frac{2}{k_{2}}=1 \text {. }
$$

Using well-known regularity properties of the Stokes system (see [32]), we obtain from (3.73) that

$$
p^{*} \psi \in L^{k_{1}}\left(0, T ; W^{2, k_{2}}(\Omega)^{N}\right)
$$

and

$$
\left\|p^{*} \psi\right\|_{L^{k_{1}}\left(W^{2, k_{2}}\right)} \leq C\left(\left\|\left(p^{*}\right)^{\prime} \psi+p^{*} p^{\prime} \varphi\right\|_{L^{k_{1}}\left(L^{k_{2}}\right)}+\|\bar{y}\|_{\infty}\left\|\nabla\left(p^{*} \psi\right)\right\|_{L^{k_{1}}\left(L^{k_{2}}\right)} .\right.
$$

We can now perform a bootstrap argument again based on a priori estimates of the Stokes system but this time with a right-hand side in $L^{k_{1}}\left(L^{\ell}\right)$ with

$$
\frac{2}{k_{1}}+\frac{6}{\ell}=1
$$


This determines the value of $k_{1}$ and, consequently, of $k_{2}$ as well. This yields $\nabla\left(p^{*} \theta\right) \in$ $L^{k_{1}}\left(L^{\ell}\right)$ and

$$
\left\|\nabla\left(p^{*} \theta\right)\right\|_{L^{k_{1}\left(L^{\ell}\right)}} \leq C\left(\left\|\left(p^{*}\right)^{\prime} \psi+p^{*} p^{\prime} \varphi\right\|_{L^{k_{1}}\left(L^{l}\right)}+\|\bar{y}\|_{\infty}\left\|\nabla\left(p^{*} \psi\right)\right\|_{L^{k_{1}\left(L^{\ell}\right)}}\right) .
$$

Combining this last inequality and (3.77), we get

$$
\begin{aligned}
\left\|\nabla\left(p^{*} \theta\right)\right\|_{L^{k_{1}\left(L^{\ell}\right)} \leq} \leq & C\left(1+\|\bar{y}\|_{\infty}^{2}\right)\left(\left\|\left(p^{*}+\left(p^{*}\right)^{\prime}\right) \Delta \psi\right\|_{L^{2}\left(L^{2}\right)}\right. \\
& +\left\|\left(\left(p^{*}+\left(p^{*}\right)^{\prime}\right) \psi\right)_{t}\right\|_{L^{2}\left(L^{2}\right)}+\left\|p^{*} p^{\prime} \Delta \varphi\right\|_{L^{2}\left(L^{2}\right)} \\
& \left.+\left\|\left(p^{*} p^{\prime} \varphi\right)_{t}\right\|_{L^{2}\left(L^{2}\right)}\right) .
\end{aligned}
$$

This tells us that we can view (3.73) as a system of $N$ heat equations with righthand sides in $L^{k_{1}}\left(0, T ; L^{\ell}(\Omega)\right)$. Then, using the representation of the solution of heat systems in terms of the semigroup, we deduce that

$$
\left\|p^{*} \psi(t)\right\|_{W^{1, \ell}(\Omega)} \leq \int_{0}^{t}(t-s)^{-1 / 2}\|B(s)\|_{L^{\ell}(\Omega)} d s \quad \forall t \in(0, T),
$$

where $B(s)=-\left(p^{*}\right)^{\prime} \psi-p^{*} p^{\prime} \varphi+D\left(p^{*} \psi\right) \bar{y}-\nabla\left(p^{*} \theta\right)$. See [40] for more details.

Since $k_{1}>2$, from Young's inequality we see that $\left\|p^{*} \psi\right\|_{W^{1, \ell}(\Omega)} \in L^{\infty}(0, T)$ and

$$
\left\|p^{*} \psi\right\|_{L^{\infty}\left(W^{1, \ell}\right)} \leq\left(1-k_{1}^{\prime} / 2\right)^{-1 / k_{1}^{\prime}} T^{-1 / 2+1 / k_{1}^{\prime}}\|B\|_{L^{k_{1}}\left(L^{\ell}\right)} .
$$

Hence, from (3.80), we obtain the estimate

$$
\begin{aligned}
\left\|p^{*} \psi\right\|_{L^{\infty}\left(W^{1, \ell}\right) \leq} & C T^{-1 / 2+1 / k_{1}^{\prime}}\left(1+\|\bar{y}\|_{\infty}^{2}\right)\left(\left\|\left(p^{*}+\left(p^{*}\right)^{\prime}\right) \Delta \psi\right\|_{L^{2}\left(L^{2}\right)}\right. \\
& \left.+\left\|\left(\left(p^{*}+\left(p^{*}\right)^{\prime}\right) \psi\right)_{t}\right\|_{L^{2}\left(L^{2}\right)}+\left\|p^{*} p^{\prime} \Delta \varphi\right\|_{L^{2}\left(L^{2}\right)}+\left\|\left(p^{*} p^{\prime} \varphi\right)_{t}\right\|_{L^{2}\left(L^{2}\right)}\right)
\end{aligned}
$$

and, consequently, with a proper choice of $\ell$, we have

$$
\begin{aligned}
\left\|p^{*} D \psi \bar{y}_{t}\right\|_{L^{2}\left(L^{q}\right)} \leq & \left\|p^{*}(D \psi)\right\|_{L^{\infty}\left(L^{\ell}\right)}\left\|\bar{y}_{t}\right\|_{L^{2}\left(L^{\sigma}\right)} \leq C\left\|\bar{y}_{t}\right\|_{L^{2}\left(L^{\sigma}\right)} T^{-1 / 2+1 / k_{1}^{\prime}}\left(1+\|\bar{y}\|_{\infty}^{2}\right) \\
& \left(\left\|\left(p^{*}+\left(p^{*}\right)^{\prime}\right) \Delta \psi\right\|_{L^{2}\left(L^{2}\right)}+\left\|\left(\left(p^{*}+\left(p^{*}\right)^{\prime}\right) \psi\right)_{t}\right\|_{L^{2}\left(L^{2}\right)}\right. \\
& \left.+\left\|p^{*} p^{\prime} \Delta \varphi\right\|_{L^{2}\left(L^{2}\right)}+\left\|\left(p^{*} p^{\prime} \varphi\right)_{t}\right\|_{L^{2}\left(L^{2}\right)}\right) .
\end{aligned}
$$

Finally, we put (3.84) together with (3.72) to obtain the desired local estimate of $\varphi_{t}$ :

$$
\begin{aligned}
& \iint_{\mathcal{O}_{4} \times(0, T)}|\widehat{p}|^{2}\left|\varphi_{t}\right|^{2} d x d t \\
& \leq\left(1+\|\bar{y}\|_{\infty}^{6}\right)\left\|\bar{y}_{t}\right\|_{L^{2}\left(L^{\sigma}\right)}^{2} e^{C\left(1+T\|\bar{y}\|_{\infty}^{2}\right)}\left(\lambda ^ { 1 2 } ( 1 + T ) \left(\|p \varphi\|_{L^{2}\left(L^{2}\left(\mathcal{O}_{1}\right)\right)}^{2}\right.\right. \\
& \left.\quad+\|p \nabla \varphi\|_{L^{2}\left(L^{2}\left(\mathcal{O}_{1}\right)\right)}^{2}+\left\|p^{\prime} \varphi\right\|_{L^{2}\left(L^{2}\left(\mathcal{O}_{1}\right)\right)}^{2}\right)+T^{-1+2 / k_{1}^{\prime}}\left(\left\|p^{*} p^{\prime \prime} \varphi\right\|_{L^{2}\left(L^{2}\right)}^{2}\right. \\
& \quad+\left\|\left(p^{*}\right)^{\prime} p^{\prime} \varphi\right\|_{L^{2}\left(L^{2}\right)}^{2}+\left\|p^{*} p^{\prime} \varphi_{t}\right\|_{L^{2}\left(L^{2}\right)}^{2}+\left\|p^{*} p^{\prime} \Delta \varphi\right\|_{L^{2}\left(L^{2}\right)}^{2} \\
& \quad+\left\|p^{*} \psi_{t}\right\|_{L^{2}\left(L^{2}\right)}^{2}+\left\|\left(p^{*}\right)^{\prime} \psi_{t}\right\|_{L^{2}\left(L^{2}\right)}^{2}+\left\|\left(p^{*}\right)^{\prime} \psi\right\|_{L^{2}\left(L^{2}\right)}^{2} \\
& \left.\left.\quad+\left\|\left(p^{*}\right)^{\prime \prime} \psi\right\|_{L^{2}\left(L^{2}\right)}^{2}+\left\|p^{*} \Delta \psi\right\|_{L^{2}\left(L^{2}\right)}^{2}+\left\|\left(p^{*}\right)^{\prime} \Delta \psi\right\|_{L^{2}\left(L^{2}\right)}^{2}\right)\right) .
\end{aligned}
$$


3.2.3. Conclusion. In this subsection we will end the proof of Lemma 3.1. We will first see that the global integrals in (3.85) can be absorbed by the terms in $I(s, \lambda ; \varphi)$, and then we will combine the local estimates to end the proof.

Notice that the next bounds hold for the weight functions:

$$
\begin{gathered}
\left|p^{*} p^{\prime}\right|+\left|\left(p^{*}\right)^{\prime} p\right| \leq C T s^{-3 / 4} \lambda^{-4} e^{-s \beta^{*}} \widehat{\gamma}^{-1 / 2} \\
\left|\left(p^{*}\right)^{\prime} p^{\prime}\right|+\left|\left(p^{*}\right)^{\prime \prime} p\right|+\left|p^{*} p^{\prime \prime}\right| \leq C T^{2} s^{1 / 4} \lambda^{-4} e^{-s \beta^{*}}\left(\gamma^{*}\right)^{3 / 4}
\end{gathered}
$$

Combining this and (3.85), we get

$$
\begin{aligned}
& \iint_{\mathcal{O}_{4} \times(0, T)}|\widehat{p}|^{2}\left|\varphi_{t}\right|^{2} d x d t \\
& \leq\left(1+\|\bar{y}\|_{\infty}^{6}\right)\left\|\bar{y}_{t}\right\|_{L^{2}\left(L^{\sigma}\right)}^{2} e^{C\left(1+T\|\bar{y}\|_{\infty}^{2}\right)}\left(\lambda ^ { 1 2 } ( 1 + T ) \left(\|p \varphi\|_{L^{2}\left(L^{2}\left(\mathcal{O}_{1}\right)\right)}^{2}\right.\right. \\
& \left.\quad+\left\|p^{\prime} \varphi\right\|_{L^{2}\left(L^{2}\left(\mathcal{O}_{1}\right)\right)}^{2}+\|p \nabla \varphi\|_{L^{2}\left(L^{2}\left(\mathcal{O}_{1}\right)\right)}^{2}\right) \\
& \quad+s^{3} \lambda^{-8} \iint_{Q} e^{-2 s \beta^{*}}\left(\gamma^{*}\right)^{3}|\varphi|^{2} d x d t \\
& \left.\quad+s^{-1} \lambda^{-8} \iint_{Q} e^{-2 s \beta^{*}} \widehat{\gamma}^{-1}\left(\left|\varphi_{t}\right|^{2}+|\Delta \varphi|^{2}\right) d x d t\right)
\end{aligned}
$$

for $s \geq C\left(T^{4}+T^{8}\right)$.

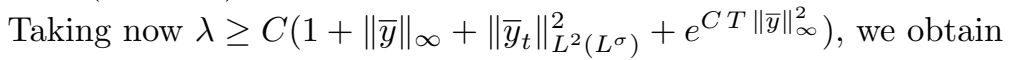

$$
\begin{aligned}
\iint_{\mathcal{O}_{4} \times(0, T)}|\widehat{p}|^{2}\left|\varphi_{t}\right|^{2} d x d t \leq C \lambda^{20}(1+T)\left(\|p \varphi\|_{L^{2}\left(L^{2}\left(\mathcal{O}_{1}\right)\right)}^{2}\right. \\
\left.+\left\|p^{\prime} \varphi\right\|_{L^{2}\left(L^{2}\left(\mathcal{O}_{1}\right)\right)}^{2}+\|p \nabla \varphi\|_{L^{2}\left(L^{2}\left(\mathcal{O}_{1}\right)\right)}^{2}\right)+\varepsilon I(s, \lambda ; \varphi) .
\end{aligned}
$$

Let us finally combine (3.89), (3.32), (3.34), and (3.59). This yields

$$
\begin{gathered}
I(s, \lambda ; \varphi) \leq C \lambda^{20}(1+T)\left(\|p \varphi\|_{L^{2}\left(L^{2}\left(\mathcal{O}_{1}\right)\right)}^{2}+\left\|p^{\prime} \varphi\right\|_{L^{2}\left(L^{2}\left(\mathcal{O}_{1}\right)\right)}^{2}\right. \\
\left.+\|p \nabla \varphi\|_{L^{2}\left(L^{2}\left(\mathcal{O}_{1}\right)\right)}^{2}\right)
\end{gathered}
$$

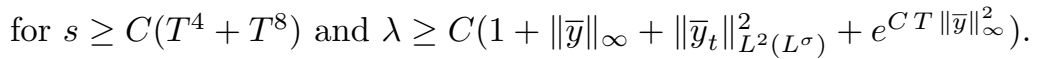

It remains only to absorb the last norm in (3.90). This can be achieved using localization arguments. Indeed, we have

$$
\begin{aligned}
\iint_{\mathcal{O}_{1} \times(0, T)}|p|^{2}|\nabla \varphi|^{2} d x d t \leq & C\left(s \lambda^{20}(1+T) \iint_{\mathcal{O} \times(0, T)} e^{2 s \beta^{*}}|p|^{4} \widehat{\gamma}|\varphi|^{2} d x d t\right. \\
& \left.+\varepsilon s^{-1} \lambda^{-20}(1+T)^{-1} \iint_{\mathcal{O} \times(0, T)} e^{-2 s \beta^{*}} \widehat{\gamma}^{-1}|\Delta \varphi|^{2} d x d t\right)
\end{aligned}
$$

for a constant $\varepsilon=\varepsilon(\Omega, \mathcal{O})>0$ small enough. 
In conclusion, we get from (3.90)

$$
I(s, \lambda ; \varphi) \leq C s^{16} \lambda^{40}\left(1+T^{2}\right) \iint_{\mathcal{O} \times(0, T)} e^{-8 s \widehat{\alpha}+6 s \alpha^{*}} \widehat{\xi}^{16}|\varphi|^{2} d x d t
$$

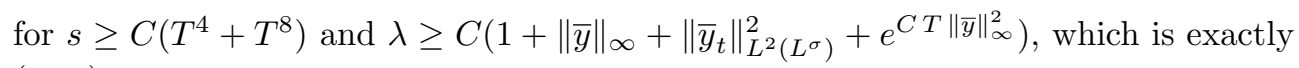
(3.13).

3.3. Some additional comments. In this subsection, we will indicate several recent results. The first two concern the controllability of the three-dimensional Stokes and Navier-Stokes systems with two scalar controls. The third one is related to the null controllability of the Stokes system with slip boundary conditions.

3.3.1. Three-dimensional Stokes and Navier-Stokes systems and two scalar controls. Let us consider system (3.7) with $N=3$. What we would like to do now is to control acting over a (small) part of $\Omega$ just in two directions. More precisely, if we denote the control by $v=\left(v_{1}, v_{2}, v_{3}\right)$, we intend to drive the system (3.7) to zero at time $t=T$ with the restriction

$$
v_{1}=0 \quad \text { or } \quad v_{2}=0 \quad \text { or } \quad v_{3}=0 .
$$

In the context of approximate controllability, this question was considered (and solved) in [49] for cylindrical domains $\Omega$.

We will have to impose an additional restriction on the control domain $\mathcal{O}$, namely,

$$
\exists x^{0} \in \partial \Omega, \exists \varepsilon>0 \text { such that } \overline{\mathcal{O}} \cap \partial \Omega \supset B\left(x^{0} ; \varepsilon\right) \cap \partial \Omega .
$$

The following result holds.

THEOREM 3.6. Let $T$ be an arbitrary positive time. Let (3.9) and (3.94) be fulfilled. Then, for every $y^{0} \in H$, there exists a two-dimensional $L^{2}$ control $v$ such that the solution to (3.7) satisfies

$$
y(T)=0 \quad \text { in } \quad \Omega .
$$

Let us indicate briefly the idea of the proof. It is not restrictive to assume that

$$
B\left(x^{0} ; \varepsilon\right) \cap \bar{\Omega} \subset \overline{\mathcal{O}}
$$

and, for instance,

$$
n_{3}(x) \neq 0 \quad \forall x \in B\left(x^{0} ; \varepsilon\right) \cap \partial \Omega .
$$

In view of the general arguments proved in section 1, the key point is to get a Carleman inequality of the form

$$
s^{-1} \iint_{Q} \rho^{2}|\varphi|^{2} d x d t \leq C \iint_{\mathcal{O} \times(0, T)} \rho^{2}\left(\left|\varphi_{1}\right|^{2}+\left|\varphi_{2}\right|^{2}\right) d x d t
$$

for the solutions to (3.10).

Let us set $\mathcal{O}_{0}=B\left(x^{0} ; \varepsilon\right) \cap \Omega$. We start from (3.13), which must hold for some $C>0$. In $\mathcal{O}_{0}$, we have

$$
\varphi_{3}(x, t)=\int_{l(x, t)}\left(\partial_{1} \varphi_{1}+\partial_{2} \varphi_{2}\right)\left(x_{1}, x_{2}, \xi_{3}, t\right) d \xi_{3},
$$

where $l(x, t)$ is an appropriate segment in $\mathcal{O}_{0}$ that starts from $(x, t)$, is parallel to $e_{3}$, and ends at a point on $\partial \Omega$. 
Using (3.97) in (3.13), in view of Fubini's formula, Hölder's inequality, and standard localization arguments, together with the particular definition of the set $\mathcal{O}_{0}$, one can find a Carleman inequality with local terms of $\left|\varphi_{1}\right|^{2}$ and $\left|\varphi_{2}\right|^{2}$ on the right-hand side. This and the dissipativity of system (3.10) imply, in a usual way, an observability inequality for the associated solutions with only these two terms on the right-hand side, which yields the result stated in Theorem 3.6.

As a consequence of Theorem 3.6, following again the arguments developed in [37] and [24], one can deduce the local exact controllability to the trajectories of the Navier-Stokes system with two scalar controls:

THEOREM 3.7. Let $(\bar{y}, \bar{p})$ be a solution of (3.15) verifying (3.9) and $\bar{y}(0) \in E=$ $H \cap L^{4}(\Omega)^{3}$. There exists $\varepsilon>0$ such that, for each $y^{0} \in E$ satisfying

$$
\left\|y^{0}-\bar{y}(0)\right\|_{L^{4}} \leq \varepsilon
$$

one can find a two-dimensional $L^{2}$ control $v$ and an associated solution $(y, p)$ to (3.14) for which we have

$$
y(T)=\bar{y}(T) \quad \text { in } \Omega .
$$

3.3.2. Stokes systems with slip boundary conditions. Let us introduce the following system:

$$
\begin{cases}y_{t}-\Delta y+\nabla p=v 1_{\mathcal{O}} & \text { in } Q \\ \nabla \cdot y=0 & \text { in } Q \\ y \cdot n=0, \quad((D y) \cdot \vec{n})_{t g}+(A(x, t) y)_{t g}=0 & \text { on } \Sigma \\ y(0)=y^{0} & \text { in } \Omega .\end{cases}
$$

Here, we have used the notation $a_{t g}$ to denote the tangential component of any vector $a \in \mathbf{R}^{N}$ :

$$
a_{t g}=a-(a \cdot n) n
$$

We will impose the following assumptions on $A$ :

$$
\begin{aligned}
& A \in H^{(3-\delta) / 2}\left(0, T ; H^{1 / 2}(\partial \Omega)^{N \times N}\right), \\
& A \in H^{1-\delta}\left(0, T ; H^{-1+N / 2+2 \delta^{\prime}}(\partial \Omega)^{N \times N}\right),
\end{aligned}
$$

where $0<\delta<1 / 2$ arbitrarily close to $1 / 2$ and $\delta<\delta^{\prime}<1 / 2$. Then we have the next result from [35].

THEOREM 3.8. Let us assume that $A$ is a uniformly elliptic matrix verifying $A \in L^{\infty}(\Sigma)^{N \times N}$ and (3.101). Then, for each $y^{0} \in H$, there exists $v \in L^{2}(\mathcal{O} \times(0, T))^{N}$ such that the solution to (3.99) satisfies

$$
y(T)=0 \quad \text { in } \Omega .
$$

Remark 10. It would be interesting to know if this result still holds, for general uniformly elliptic $A \in L^{\infty}(\Sigma)^{N \times N}$, without the hypotheses (3.101). However, this seems to be a difficult question.

For similar systems for the Stokes or Navier-Stokes equations involving nonlinear slip boundary conditions, the situation is much more complicated. See [35] for more details. 


\section{REFERENCES}

[1] V. M. Alekseev, V. M. Tikhomirov, and S. V. Fomin, Optimal Control, Contemp. Soviet Math., Consultants Bureau, New York, 1987.

[2] S. ANITA AND V. BARBU, Null controllability of nonlinear convective heat equations, ESAIM Control Optim. Calc. Var., 5 (2000), pp. 157-173.

[3] J.-P. Aubin, L'analyse non linéaire et ses motivations économiques, Masson, Paris, 1984.

[4] S. A. Avdonin And S. A. Ivanov, Families of Exponentials. The Method of Moments in Controllability Problems for Distributed Parameter Systems, Cambridge University Press, Cambridge, UK, 1995.

[5] V. BARBU, Exact controllability of the superlinear heat equation, Appl. Math. Optim., 42 (2000), pp. $73-89$.

[6] V. BARbu, Controllability of parabolic and Navier-Stokes equations, Sci. Math. Jpn., 56 (2002), pp. 143-211.

[7] V. Barbu And M. IAnneldi, Controllability of the heat equation with memory, Differential Integral Equations, 13 (2003), pp. 1393-1412.

[8] V. Barbu And G. Tessitore, Null controllability of stochastic heat equations with a multiplicative noise, Appl. Math. Optim., 47 (2000), pp. 97-120.

[9] O. Bodart, M. González-Burgos, and R. PÉrez-García, Insensitizing controls for a semilinear heat equation with a superlinear nonlinearity, C. R. Math. Acad. Sci. Paris, 335 (2002), pp. 677-682.

[10] J.-M. Coron, On the controllability of the 2-D incompressible Navier-Stokes equations with the Navier slip boundary conditions, ESAIM Control Optim. Calc. Var., 1 (1996), pp. 35-75.

[11] J.-M. Coron And A. V. Fursikov, Global exact controllability of the 2-D Navier-Stokes equations on a manifold without boundary, Russian J. Math. Phys., 4 (1996), pp. 1-19.

[12] L. DE TERESA, Insensitizing controls for a semilinear heat equation, Comm. Partial Differential Equations, 25 (2000), pp. 39-72.

[13] A. Doubova And E. Fernández-CARA, Some control results for simplified one-dimensional models of fluid-solid interaction, Math. Models Methods Appl. Sci., 15 (2005), pp. 783-824.

[14] A. Doubova, E. Fernández-Cara, and M. GonzÁlez-Burgos, Controllability results for linear viscoelastic fluids of the Maxwell and Jeffreys kinds, C. R. Acad. Sci. Paris, 331 (2000), pp. 537-542.

[15] A. Doubova, E. Fernández-Cara, and M. González-Burgos, On the controllability of the heat equation with nonlinear boundary Fourier conditions, J. Differential Equations, 196 (2004), pp. 385-417.

[16] A. Doubova, E. Fernández-Cara, M. González-Burgos, and E. Zuazua, On the controllability of parabolic systems with a nonlinear term involving the state and the gradient, SIAM J. Control Optim., 41 (2002), pp. 798-819.

[17] Yu. V. Egorov, Some problems in the theory of optimal control, Z. Vycisl. Mat. i Mat. Fiz., 3 (1963), pp. 887-904 (in Russian).

[18] C. FABRE, Uniqueness results for Stokes equations and their consequences in linear and nonlinear control problems, ESAIM Control Optim. Calc. Var., 1 (1995/96), pp. 267-302.

[19] C. Fabre, J.-P. Puel, and E. Zuazua, Approximate controllability of the semilinear heat equation, Proc. Roy. Soc. Edinburgh Sect. A, 125 (1995), pp. 31-61.

[20] E. FERnÁNDEZ-CARA, Null controllability of the semilinear heat equation, ESAIM Control Optim. Calc. Var., 2 (1997), pp. 87-103.

[21] E. Fernández-Cara, M. González-Burgos, S. Guerrero, and J.-P. Puel, Null controllability of the heat equation with Fourier Fourier boundary conditions: The linear case, ESAIM Control Optim. Calc. Var., 12 (2006), pp. 442-465.

[22] E. Fernández-Cara, M. González-Burgos, S. Guerrero, and J.-P. Puel, Exact controllability to the trajectories of the heat equation with Fourier boundary conditions: The semilinear case, ESAIM Control Optim. Calc. Var., 12 (2006), pp. 466-483.

[23] E. Fernández-CARA AND S. Guerrero, Global Carleman estimates for solutions of parabolic systems defined by transposition and some applications to controllability, AMRX Appl. Math. Res. Express, Volume 2006 (2006), article 75090; available online from http://www.hindawi.com/GetArticle.aspx?doi=10.1155/AMRX/2006/75090.

[24] E. Fernández-Cara, S. Guerrero, O. Yu. Imanuvilov, and J.-P. Puel, Local exact controllability to the trajectories of the Navier-Stokes equations, J. Math. Pures Appl., 83 (2004), pp. 1501-1542.

[25] E. Fernández-Cara, S. Guerrero, O. Yu. Imanuvilov, and J.-P. Puel, Some controllability results for the $N$-dimensional Navier-Stokes and Boussinesq systems with $N-1$ scalar controls, SIAM J. Control Optim., 45 (2006), pp. 146-173. 
[26] E. Fernández-CARA And L. DE Teresa, Null controllability of a cascade system of parabolichyperbolic equations, Discrete Contin. Dyn. Syst., 11 (2004), pp. 699-714.

[27] E. Fernández-Cara And E. Zuazua, The cost of approximate controllability for heat equations: The linear case, Adv. Differential Equations, 5 (2000), pp. 465-514.

[28] E. Fernández-Cara And E. Zuazua, Null and approximate controllability for weakly blowing up semilinear heat equations, Ann. Inst. H. Poincaré Anal. Non Linéaire, 17 (2000), pp. 583-616.

[29] A. V. Fursikov and O. Yu. Imanuvilov, Local exact controllability of the Navier-Stokes equations, C. R. Acad. Sci. Paris Sér. I Math., 323 (1996), pp. 275-280.

[30] A. V. Fursikov and O. Yu. Imanuvilov, Controllability of Evolution Equations, Lecture Note Series 34, Research Institute of Mathematics, Seoul National University, Seoul, 1996.

[31] A. V. Fursikov and O. Yu. Imanuvilov, Exact controllability of the Navier-Stokes and Boussinesq equations, Uspekhi Mat. Nauk, 54 (1999), pp. 93-146 (in Russian); English translation in Russian Math. Surveys, 54 (1999), pp. 565-618.

[32] Y. Giga And H. Sohr, Abstract $L^{p}$ estimates for the Cauchy problem with applications to the Navier-Stokes equations in exterior domains, J. Funct. Anal., 102 (1991), pp. 72-94.

[33] O. GLass, An addendum to a J. M. Coron theorem concerning the controllability of the Euler system for $2 D$ incompressible inviscid fluids, J. Math. Pures Appl. (9), 80 (2001), pp. 845877.

[34] R. GLOWinski AND J.-L. Lions, Exact and approximate controllability for distributed parameter systems, in Acta Numerica, Cambridge University Press, Cambridge, UK, 1995, pp. 159333.

[35] S. Guerrero, Local exact controllability to the trajectories of the Navier-Stokes system with nonlinear Navier-slip boundary conditions, ESAIM Control Optim. Calc. Var., 12 (2006), pp. 484-544.

[36] O. Yu. Imanuvilov, Controllability of parabolic equations, Sb. Math., 186 (1995), pp. 109-132 (in Russian).

[37] O. Yu. Imanuvilov, Remarks on exact controllability for the Navier-Stokes equations, ESAIM Control Optim. Calc. Var., 6 (2001), pp. 39-72.

[38] O. Yu. Imanuvilov and J.-P. Puel, Global Carleman estimate for weak elliptic non homogeneous Dirichlet problem, Int. Math. Res. Not., 16 (2003), pp. 883-913.

[39] O. Yu. Imanuvilov And M. Yamamoto, Carleman estimate for a parabolic equation in a Sobolev space of negative order and its applications, in Control of Nonlinear Distributed Parameter Systems, Lecture Notes in Pure and Appl. Math. 218, Marcel Dekker, New York, 2001, pp. 113-137.

[40] O. A. Ladyzhenskaja, V. A. Solonnikov, and N. N. Ural'ceva, Linear and Quasilinear Equations of Parabolic Type, translated from the Russian by S. Smith, Translations of Mathematical Monographs 23, AMS, Providence, RI, 1967.

[41] G. Lebeau and L. Robbiano, Contrôle exact de l'équation de la chaleur, Comm. Partial Differential Equations, 20 (1995), pp. 335-356.

[42] G. Lebeau and E. Zuazua, Null-controllability of a system of linear thermoelasticity, Arch. Rational Mech. Anal., 141 (1998), pp. 297-329.

[43] G. Lebeau and E. Zuazua, Decay rates for the three-dimensional linear system of thermoelasticity, Arch. Rational Mech. Anal., 148 (1999), pp. 179-231.

[44] J.-L. Lions, Exact controllability, stabilizability and perturbations for distributed systems, SIAM Rev., 30 (1988), pp. 1-68.

[45] J.-L. Lions, Contrôlabilité Exacte, Perturbations et Stabilisation de Systèmes Distribués, Tome 1, Rech. Math. Appl. 8, Masson, Paris, 1988.

[46] J.-L. Lions, Contrôlabilité Exacte, Perturbations et Stabilisation de Systèmes Distribués, Tome 2, Rech. Math. Appl. 9, Masson, Paris, 1988.

[47] J.-L. Lions, Quelques notions dans l'analyse et le contrôle de systèmes à données incomplètes, in Proceedings of the 11th Congress on Differential Equations and Applications/First Congress on Applied Mathematics, University of Málaga, Málaga, 1989, pp. 43-54.

[48] J.-L. Lions, Are there connections between turbulence and controllability?, in Analysis and Optimization of Systems, A. Bensoussan and J.-L. Lions, eds., Lecture Notes Control and Inform. Sci. 144, Springer-Verlag, Berlin, 1990.

[49] J.-L. Lions and E. Zuazua, A generic uniqueness result for the Stokes system and its control theoretical consequences, in Partial Differential Equations and Applications, P. Marcellini, G. Talenti, and E. Visentini, eds., Lecture Notes in Pure and Appl. Math. 177, Marcel Dekker, New York, 1996, pp. 221-235.

[50] J.-L. Lions AND E. ZuazuA, Exact boundary controllability of Galerkin's approximations of Navier-Stokes equations, Ann. Scuola Norm. Sup. Pisa Cl. Sci. (4), 26 (1998), pp. 605-621. 
[51] J.-P. Puel, Controllability of Partial Differential Equations, Lectures in the Universidade Federal do Rio de Janeiro, Brazil, 2002.

[52] D. L. Russell, A unified boundary controllability theory for hyperbolic and parabolic partial differential equations, Int. Math. Res. Not., 52 (1973), pp. 189-221.

[53] D. L. Russell, Controllability and stabilizability theory for linear partial differential equations: Recent progress and open questions, SIAM Rev., 20 (1978), pp. 639-739.

[54] Th. I. Seidman, On uniform null-controllability and blow-up estimates, in Control Theory of Partial Differential Equations, Lect. Notes Pure Appl. Math. 242, Chapman \& Hall/CRC, Boca Raton, FL, 2005, pp. 213-227.

[55] R. Temam, Navier-Stokes Equations. Theory and Numerical Analysis, 2nd ed., North-Holland, Amsterdam, 1977.

[56] E. ZuAzUA, Exact boundary controllability for the semilinear wave equation, in Nonlinear Partial Differential Equations and Their Applications, Vol. 10, Pitman Res. Notes Math. Ser. 220, Longman, Harlow, UK, 1991, pp. 357-391.

[57] E. Zunzua, Controllability of the linear system of thermoelasticity, J. Math. Pures Appl., 74 (1995), 303-346. 